\title{
Analysis of the Torsion-Rotation Spectra of the Isotopic Methanol Molecules
}

\author{
Yuen Yuen Kwan and David M. Dennison \\ Department of Physics, University of Michigan, Ann Arbor, Michigan, 48104
}

The high resolution infrared spectra of $\mathrm{CH}_{3} \mathrm{OH}, \mathrm{CH}_{3} \mathrm{OD}, \mathrm{CD}_{3} \mathrm{OH}$ and $\mathrm{CD}_{3} \mathrm{OD}$ observed by D. R. Woods and C. W. Peters in the region from 400 to $900 \mathrm{~cm}^{-1}$ have been analyzed to obtain the molecular moments of inertia, the barrier height and the Kirtman perturbation constants. The first step consisted in identifying as many $Q$ branch origins as possible. Between 25 and 40 origins were determined for each isotopic molecule with an accuracy of about $0.03 \mathrm{~cm}^{-1}$. These data were combined with $Q$ branch origins found by $R$. M. Lees and J. G. Baker from their very accurate measurements of the microwave spectra of these molecules. A nonlinear least squares solution yielded values for the constants. With these constants the spectrum was recomputed and found to agree with the observed spectrum to the order of the experimental errors. In all, 176 dala points were described by 40 constants -10 for each molecule.

In the second phase of the work a tentative theory is proposed which relates the perturbation constants of the four isotopic molecules. This theory, which contains a number of approximations, involves only 21 constants. These were evaluated from a nonlinear least squares analysis and the spectrum was recomputed. The agreement, with the ohserved speetrum is good, but not as good as in the former case.

The barrier height $V_{3}$ was found to decrease upon deuteration and a mechanism is suggested based upon the zero point vibrations of the atoms. The barrier potential is shown to be highly sinusoidal in form with $V_{6} / V_{3}=$ $-0.0002 \pm 0.0006$.

\section{I, INTRODUCTION}

Recently two important advances have been made towards a better understanding of the torsion-rotation spectra of the methanol molecules $\mathrm{CH}_{3} \mathrm{OH}$, $\mathrm{CH}_{3} \mathrm{OD}, \mathrm{CD}_{3} \mathrm{OH}$ and $C D_{3} \mathrm{OD}$. The first of these is contained in papers by $\mathrm{R} . \mathrm{M}$. Lees and J. G. Baker (1) and by R. M. Lees (2) while the second consists of high resolution measurements by D. R. Woods and C. W. Peters (3) of the infrared spectra of these molecules in the general region from around 400-900 $\mathrm{cm}^{-1}$.

Lees and Baker determined the microwave spectra of the several methanols from 90 to $200 \mathrm{GHz}$. Their analysis employed, with certain important modifi- 
cations, the methods developed by earlier investigators (4-8). These methods had concentrated on expressing the energy levele as a function of the moments and products of inertia together with the height of a sinusoidal hindering potential barrier. Certain of the more important perturbations due to centrifugal distortions were included but not on a completely systematic basis. Lees and Baker extended the earlier analyses by introducing the full set of perturbation terms suggested by Kirtman (9). These perturbation terms may be divided into three groups: (a) the first order deviation from a sinusoidal potential $1 / 2 V_{6}(1-\cos 6 \gamma)$ where $\gamma$ is the angle of internal rotation, (b) the analog of the usual centrifugal distortion terms which are quartic in the expectation values of the several angular momenta, and (c) the effect of the centrifugal distortions on the effective height of the potential barrier. These terms which involve 14 semiempirical constants will be described in more detail in later sections.

Lees and Baker found that their microwave lines by themselves were not very suitable for determining the full set of Kirtman constants in spite of the high accuracy of the experimental measurements. The reason for this was that most of the microwave lines in question involved transitions between states with torsional quantum number $n=0$, while the contributions from many of the Kirtman tcrms only become significant for larger values of $n$. However, by combining their data with the measurements of the infrared spectrum of $\mathrm{CH}_{3} \mathrm{OH}$ obtained by Burkhard and Dennison ( 8 ) they were able to calculate a set of perturbation constants which would reproduce satisfactorily all the known lines. Burkhard and Dennison had measured the torsion-rotation spectrum from 50 to $460 \mathrm{~cm}^{-1}$ where most of the lines come from transitions to the states of torsional numbers $n=1$ and 2 . Unfortunately, single lines in the infrared spectrum were not resolved and only the $Q$ branches with their unknown convergences could be measured. The overall accuracy was estimated to be $0.5 \mathrm{~cm}^{-1}$. Moreover, the infrared rotation spectra of the deuterated methanols had never been measured.

A second limitation which was discovered by Lees and Baker stems from the fact that there exists a relationship between certain of the matrix elements which occur as multipliers of the Kirtman constants in the energy expression. This means that an infinity of sets of these particular constants will equally well reproduce the experimental data. Unfortunately one of the most interesting constants, namely $V_{6}$, is involved and consequently its value could not be determined uniquely.

The second important advance referred to arises from the experimental work of Woods and Peters on the torsion-rotation spectra of the methanols, normal and deuterated. The resolution they obtained was sufficiently high so that a great number of individual lines of the $P$ and $R$ branches could be measured. The resulting accuracy of determining the $Q$ origins was about $0.03 \mathrm{~cm}^{-1}$. More- 
over, the spectra were extended to higher frequencies with the result that states with torsional numbers of $n=3$ and 4 could be found.

The program of the present investigation may be divided into three parts of which the first is to identify as many of the lines found by Woods and Peters as possible. The second part consisted in making a least squares fit of the experimental data to determine the molecular parameters, including the Kirtman constants, for each of the four methanols. As will be seen the results of this calculation allow a theoretical determination of any of the energy levels in the range $n=0-4$ and $K=0-15$ with an accuracy which is of the order of the experimental errors. Unfortunately for the reason cited above this procedure does not allow a unique evaluation of $V_{6}$.

The final portion of the program describes a tentative and approximate theory which attempts to interrelate the Kirtman constants of all four methanols and which avoids the ambiguity present in fitting the constants to the data for any one of the methanols. Since only about half as many constants are required for the fitting of all the experimental data, the accuracy of the fit is somewhat less but it is believed that the physical significance of the constants is greater.

\section{THEORETICAL}

\section{The Energy Levels}

In the main we will employ the methods developed by the earlier investigators. We shall, with a few exceptions, use the notation of Lees and Baker. The calculation starts by considering a rigid symmetrical model consisting of two parts, representing the methyl group and the hydroxyl group, which can rotate with respect to each other subject to a threefold hindering potential. The Hamiltonian may be separated into two parts,

$$
H^{0}=H_{\text {sym }}^{0}+H_{T}{ }^{0}
$$

where $H_{R \text { sym }}^{0}$ is the Hamiltonian of a symmetrical rotator with effective moments of inertia to be defined shortly and $H_{T}{ }^{0}$ describes the hindered rotation between the two parts of the molecule.

The wavefunctions of $H_{R \text { sym }}^{0}$ are just those of a symmetrical rotator and are expressed in terms of modified Eulerian angles describing the molecule as a whole. (See, for example, Hecht and Dennison ( $(7)$ ).

$$
U_{R}{ }^{0}=\frac{1}{2 \pi} \Theta_{J K M}(\theta) e^{i M \psi} e^{i K \varphi} .
$$

The quantum numbers $J, K$ and $M$ measure, respectively, the total angular momentum, its component along the symmetry axis of the molecule and its

${ }^{1}$ Lees and Baker include the asymmetric contribution $H_{R_{\mathrm{asym}}}^{0}$ in their zeroth order Hamiltonian but since this is small for the methanol molecule, we prefer to consider it as a perturbation. 
component along some space fixed axis. The matrix representing $H_{R \text { sym }}^{0}$ is diagonal and has the elements

$$
\left(E_{R}{ }^{0}\right) / h c=\frac{1}{2}(B+C)\left(J^{2}+J\right)+\left[A-\frac{1}{2}(B+C)\right] K^{2} .
$$

The constants $A, B$ and $C$, as well as the constants $D_{a b}$ and $F$ which will be introduced shortly are related to the moments and products of inertia as follows ${ }^{2}$

$$
\begin{aligned}
A & =\left[\left(I_{a}+I_{b}\right) /\left(I_{a} I_{b}-I_{a b}^{2}\right)-I_{b} /\left(I_{b}^{2}+I_{a b}^{2}\right)\right] \hbar / 4 \pi \mathrm{c}, \\
B & =\left[I_{b} /\left(I_{b}^{2}+I_{a b}^{2}\right)\right] \hbar / 4 \pi \mathrm{c}, \\
C & =\left[1 / I_{c}\right) \hbar / 4 \pi \mathrm{c}, \\
D_{a b} & =\left[I_{a b} /\left(I_{b}^{2}+I_{a b}^{2}\right)\right] \hbar / 4 \pi \mathrm{c}, \\
F & =\left[\left(I_{a} I_{b}-I_{a b}^{2}\right) /\left(I_{a 1} I_{a 2} I_{b}-I_{a 2} I_{a b}^{2}\right)\right] \hbar / 4 \pi \mathrm{c} .
\end{aligned}
$$

The inertial tensor $I$ is calculated in a molecular axis system with origin at the center of mass of the molecule, with $a$ axis parallel to the axis of internal rotation (assumed to be the axis of the methyl group) and with $c$ axis perpendicular to the $\mathrm{COH}$ plane. $I_{a 2}$ is the moment of inertia of the methyl group about its symmetry axis and $I_{a 1}$ is defined by the relation $I_{a}=I_{a 1}+I_{a 2} . I_{a b}$ is the pronuct of inertia about the $a$ and $b$ axes.

The torsional part of the Hamiltonian is,

$$
\left(H_{T}^{0}\right) / h c=F P_{\gamma}^{2}+\frac{1}{2} V_{3}(1-\cos 3 \gamma),
$$

where $\gamma$ is angle of internal rotation between the methyl and hydroxyl groups and $P_{\gamma}=(1 / i) \delta / \delta \gamma$. The resulting wave equation is a Mathicu equation and the eigenvalues are determined by imposing single valuedness conditions. The wave equation may be solved as pointed out by Koehler and Dennicon (4) through the use of continued fractions. It may also be solved by diagonalizing the matrix for $H_{T}{ }^{0}$ using a free internal rotator representation. Lees and Baker employ the first method while we have elected to use the second. The second method necessitates diagonalizing an infinite matrix but we find that by truncating it to a $21 \times 21$ matrix we introduce no appreciable errors.

The eigenvalues of the torsional wavefunction, namely, $E_{n \tau K}$ depend upon the three quantum numbers $n, \tau$ and $K$, where $n$ measures the torsion. In the limit of very high barriers the energy would have the approximate form $h \nu_{T}(n+1 / 2)$ where $\nu_{T}$ is the torsional frequency. The number $\tau$ takes on the values 1,2 or 3 and arises from the threefold nature of the hindering potential. The form of $E_{n \tau K}$ for $\mathrm{CH}_{3} \mathrm{OH}$ is plotted in Fig. 1 of Ref. (8). For given values

2 Our notation differs slightly from that of Lees and Baker in that we express our constants in waves per centimeter rather than in $\mathrm{MHz}$. 
of $n$ and $\tau$ the curves appear to be periodic analytical functions of $K$ although of course only the discrete points $K=0,1,2, \cdots$ have a physical meaning.

Lees and Baker do not use the quantum number $\tau$ but instead, following Lin and Swalen $(10)$, employ the three symmetry species $A, E_{1}$, and $E_{2}$. There is much to be said for this choice since the selection rules impose the simple condition that transitions can only occur between levels of the same symmetry species. For the $\tau$ choice, the selection rules appear to be more complicated, thus if $K \rightarrow K-1 ; \tau=1 \rightarrow 2,2 \rightarrow 3$ or $3 \rightarrow 1$ and vice versa. On the other hand the energy levels-including perturbation terms-are smooth functions of $K$ for any given values of $n$ and $\tau$. We have found that this property has been very valuable in extrapolating from known lines of the experimental spectrum for the purpose of identifying as yet unknown lines. In any case these are two equally valid systems of notation and the connection between them is the following: The $A$ states are those for which $K+\tau=3 N+1$, the $E_{1}$ states have $K+\tau=3 N$ and the $E_{2}$ states have $K+\tau=3 N+2$ where $N$ is any integer.

The wavefunctions for the torsional Hamiltonian $H_{T}{ }^{0}$ have the form

$$
U_{n ; K}^{0}(\gamma)=\exp \left(i_{\rho} K \gamma\right) P_{n \tau K},
$$

where

$$
\rho=\left[I_{a 2}\left(I_{b}^{2}+I_{a b}^{2}\right)^{1 / 2} /\left(I_{a} I_{b}-I_{a b}^{2}\right)\right]-1,
$$

and

$$
P_{n \tau K}=(2 \pi)^{-1 / 2} \sum_{m} a_{n+K m} \exp (i m \gamma) .
$$

It is convenient to normalize $V_{n, R}^{0}$ and this is accomplished hy choosing a multiplier for the $a_{n+K m}$ such that

$$
\sum_{m} a_{n+K m}^{2}=1 .
$$

$m$ is a running index which takes on the values $3 l$ for $\tau=1$ states, $3 l-1$ for $\tau=2$ and $3 l+1$ for $\tau=3$, where $l$ is any integer.

The next step consists in calculating the various perturbation terms using as basis eigenfunctions $U_{R}{ }^{0} U_{n T K}^{0}$. The first of these terms we take to be the contribution due to the asymmetry of the molecule. The part of the Hamiltonian describing these is labeled $H_{R \text { asym }}^{0}$ by Lees and Baker. It has no diagonal elements but it does have the following off-diagonal elements:

$$
\begin{aligned}
&\left\langle n^{\prime} \tau^{\prime} J K+1\left|H_{R \text { asym }}^{0}\right| n_{\tau} J K\right\rangle= \frac{1}{2} D_{a b}(2 K+1) \\
& \cdot[(J-K)(J+K+1)]^{1 / 2} I_{1} \\
&\left\langle n^{\prime} \tau^{\prime} J K+2\left|H_{R \text { a } y \text { ym }}^{0}\right| n_{\tau} J K\right\rangle=\frac{1}{4}(B-C)[(J-K)(J-K-1) \\
&\cdot(J+K+1)(J+K+2)]^{1 / 2} I_{2},
\end{aligned}
$$


where

$$
\begin{aligned}
& I_{1}=\int_{0}^{2 \pi} P_{n \tau K}^{*} e^{-i \gamma} P_{n^{\prime} \tau^{\prime} K+1} d \gamma \\
& I_{2}=\int_{0}^{2 \pi} P_{n \tau K}^{*} e^{-2 i \gamma} P_{n^{\prime} \tau^{\prime} K+2} d \gamma
\end{aligned}
$$

In the above equations the allowed $\tau$ connections are such that the initial and final states belong to the same symmetry species $A, E_{1}$, or $E_{2}$.

The states of symmetry species $E_{1}$ and $E_{2}$ are doubly degenerate and this degeneracy is not removed by any perturbation having a threefold symmetry in the torsion angle $\gamma$. The asymmetric terms in the Hamiltonian, however, shift these levels by an amount,

$$
D_{n \tau K J}=d_{0}+\left(J^{2}+J\right) d_{1}+\left(J^{2}+J\right)^{2} d_{2},
$$

where the quantities $d_{0}, d_{1}$ and $d_{2}$ are functions of $n, \tau$ and $K$. They may be calculated by standard perturbation methods which in our case have been carried through second order. The asymmetry of the methanol molecules is quite small (even for $\mathrm{CD}_{3} \mathrm{OD}$ ) and as a result $D_{n \tau K J}$ is a relatively small quantity which is seldom larger than $0.1 \mathrm{~cm}^{-1}$.

The states of symmetry species $A$ are also doubly degenerate for the Hamiltonian $H^{0}$ (with the exception of the states where $K=0$ ) but this degeneracy is removed by the asymmetric term. Again the correction to the energy may be calculated by standard perturbation methods which lead to the expression, $D_{n \tau K J} \pm S_{n \tau K J}$ where $D_{n \tau K J}$ represents the shift of the center of gravity of the levels and $2 S_{n \tau K J}$ gives their splitting. The wavefunctions for these two levels and the associated selection rules have been described in detail by Ivash and Dennison (6). $S_{n T K J}$ decreases very rapidly with increasing $K$ and for the methanol molecules the only states which need be considered are $K=1$ or 2 . For $K=1, S$ is proportional to $J^{2}+J$ and for the larger obscrved values of $J$ may be of the order of several waves per centimeter. The contributions of $S$ for the $K=2$ states contain terms proportional to $J^{2}+J$ and to $\left(J^{2}+J\right)^{2}$ but the splitting is generally less than $0.1 \mathrm{~cm}^{-1}$. It will be noted that the perturbation terms arising from $H_{R \text { asym }}^{0}$ involve only inertial constants and the barrier height $V_{3}$.

The remaining perturbation terms contain the semiempirical Kirtman constants. The first of these expresses the deviation of the hindering potential from a sinusoidal form and is equal to $1 / 2 V_{6}(1-\cos 6 \gamma)$. First order perturbation theory yields $\frac{1}{2} V_{6}\langle(1-\cos 6 \gamma)\rangle$ as its contribution to the energy where of course the eigenfunctions of $H_{T}{ }^{0}$ are used in calculating the average value of $(1-\cos 6 \gamma)$. Note that this average value will be a function of $n, \tau$ and $K$ but not of $J$. 
The next set of terms are quartic in the several angular momenta and result in the following addition to the energy:

$$
\begin{aligned}
& G_{v}\left(J^{2}+J\right)\left\langle P_{\gamma}^{2}\right\rangle+L_{v}\left(J^{2}+J\right) K\left\langle P_{\gamma}\right\rangle-D_{J J}\left(J^{2}+J\right)^{2} \\
& -D_{J K}\left(J^{2}+J\right) K^{2}-D_{K K} K^{4}+k_{1} K^{3}\left\langle P_{\gamma}\right\rangle+k_{2} K^{2}\left\langle P_{\gamma}^{2}\right\rangle \\
& +k_{3} K\left\langle P_{\gamma}^{3}\right\rangle+k_{4}\left\langle P_{\gamma}^{4}\right\rangle .
\end{aligned}
$$

These terms may be considered as an extension of the centrifugal distortion terms $D_{J J}, D_{J K}$ and $D_{K K}$, for a symmetrical rotator without internal rotation to the case of a symmetrical rotator possessing internal hindered rotation. Again the average values of the various powers of $P_{\gamma}$ will depend upon $n, \tau$, and $K$ but not upon $J$. A simple calculation yields the result $\left\langle P_{\gamma}{ }^{8}\right\rangle=\sum_{m}(m+\rho K)^{8} a_{n \tau K m}^{2}$, where $s$ is a positive integer.

The final set of terms describes the effect of the centrifugal distortion on the effective height of the barrier $V_{3}$. They are

$$
\begin{aligned}
& F_{v}\left(J^{2}+J\right)\langle 1-\cos 3 \gamma\rangle+k_{5} K^{2}\langle 1-\cos 3 \gamma\rangle \\
& \quad+k_{6} K\left\langle P_{\gamma}(1-\cos 3 \gamma)\right\rangle+k_{7}\left\langle P_{\gamma}^{2}(1-\cos 3 \gamma)\right\rangle .
\end{aligned}
$$

Lees and Baker let $k_{6}$ be the coefficient of $K\left\langle P_{\gamma}\right\rangle$ but the two expressions are identical since it can be shown that $\left\langle P_{\gamma} \cos 3 \gamma\right\rangle=0$.

\section{The Redundancy Relation}

Lees and Baker in their initial attempt towards fitting the experimental frequencies of $\mathrm{CH}_{3} \mathrm{OH}$ with the above energy expression (including the Kirtman terms) found that their nonlinear least squares solution diverged and would not yield convergent values for the constants. They discovered that the difficulty arose from the existence of a relationship, which we shall call a redundancy relationship, between certain of the matrix elements associated with the Hamiltonian $H_{T}{ }^{0}$, namely,

$$
\begin{aligned}
\frac{9}{4}\langle 1-\cos 3 \gamma\rangle-\left(V_{3} / 8 F\right)\langle 1 & -\cos 6 \gamma\rangle \\
& +\left\langle P_{\gamma}{ }^{2}\right\rangle-\left\langle P_{\gamma}{ }^{2}(1-\cos 3 \gamma)\right\rangle=\frac{9}{4} .
\end{aligned}
$$

It follows that a number of the terms in the energy expression are not independent but are connected through the redundancy relation. Thus it is not possible uniquely to determine the constants in question, namely, $I_{a 1}, I_{a 2}, V_{3}, V_{6}, k_{6}$ and $k_{7}$. Actually if the perturbation terms are set equal to zero, one can fit the observed spectrum moderately well with the large constants $I_{a 1}, I_{a 2}$, and $V_{3}$. It is only when we introduce the small constants, $V_{6}, k_{6}$ and $k_{7}$ that the difficulty appears. It is therefore correct to say that the observed spectrum of any one of 
the methanols can not uniquely determine the constants $V_{6}, k_{6}$, and $k_{7}$ nor fix $I_{a 1}, I_{a 2}$, and $V_{3}$ within certain small limits of uncertainty. One way of stating the consequences of the redundancy relation is that an infinity of sets of the constants in question will lead to the same energy levels for the molecule, at least to the order of approximation to which we are working. We have explicitly calculated the equations connecting two such sets of constants. They are as follows where we have retained only the linear terms in the expansion of the zeroth order energy.

$$
\begin{aligned}
I_{a 1}^{\prime}+N_{1} k_{7}^{\prime} & =I_{a 1}^{\prime \prime}+N_{1} k_{7}^{\prime \prime}, \\
I_{a 1}^{\prime}+I_{a 2}^{\prime} & =I_{a 1}^{\prime \prime}+I_{a 2}^{\prime \prime}, \\
2 V_{3}^{\prime}+9 k_{7}^{\prime} & =2 V_{3}^{\prime \prime}+9 k_{7}^{\prime \prime}, \\
V_{6}^{\prime}-\left(V_{3} / 4 F\right) k_{7}^{\prime} & =V_{6}^{\prime \prime}-\left(V_{3} / 4 F\right) k_{7}^{\prime \prime}, \\
k_{6}{ }^{\prime}+N_{2} k_{7}^{\prime} & =k_{6}^{\prime \prime}+N_{2} k_{7}^{\prime \prime},
\end{aligned}
$$

where

$$
\begin{aligned}
& N_{1}=(4 \pi c / \hbar)\left[1 / I_{a 2}^{2}-I_{b}^{2} /\left(I_{a 1} I_{b}-I_{a b}^{2}\right)^{2}\right]^{-1}, \\
& N_{2}=(\hbar / 2 \pi c)\left[\left(I_{b}{ }^{2}+I_{a b}^{2}\right)^{1 / 2} / I_{a 2}\left(I_{a 1} I_{b}-I_{a b}^{2}\right)\right] N_{1} .
\end{aligned}
$$

If any one of the constants $V_{6}, k_{6}$, or $k_{7}$ were known (which they are not) it would be possible to fit the observed data by least squares and obtain all the remaining constants. Lees and Baker in their treatment of $\mathrm{CH}_{3} \mathrm{OH}$ have made three arbitrary choices, namely, $k_{6}=0, k_{7}=0$ or $V_{6}=0$ and these they label respectively Fits $I, I I$, and III. The experimental data are equally well reproduced by each fit as of course they must be. As a test of our equations we have calculated the relationships between the constants $I_{a 1}, I_{a 2}, V_{3}, V_{6}, k_{6}$, and $k_{7}$ for each pair of fits and find that they are satisfied to within experimental error. In our analyses of the spectra of each of the methanols we have elected to employ Fit II where $k_{7}=0$. There was no physical reason for this choice and in fact it will appear in the last section of our work that Fit III more nearly describes the actual situation. However, for the purpose of calculating energy levels there is no disadvantage in letting $k_{7}=0$.

\section{Intensities}

In attempting to identify the lines in the crowded and complex spectra observed by Woods and Peters it is essential to calculate the intensities of the lines which may be expected to fall within the region in question. It will be sufficient for our purpose to employ a zerothorder approximation, that is, one which uses the wavefunctions of a system with Hamiltonian $H^{0}$. The general formula for the integrated intensity of an absorption line resulting from a transition from the 
$i$-th to the $j$-th state is well known (8). In our case the expression becomes

$$
I_{j_{+} i}=A \nu f(i|M| j)^{2}[1-\exp (-h \nu / k T)] \exp (-E i / k T) .
$$

$A$ is a proportionality constant and $\nu$ is the frequency of the transition. $f$ is a weight factor arising from the spin of the protons (or the deuterons) in the methyl group. In the case of $\mathrm{CH}_{3} \mathrm{OH}$ and $\mathrm{CH}_{3} \mathrm{OD}$ the three equivalent protons with their spins of $1 / 2$ and overall antisymmetric statistics impose the condition that $f=2$ for states of either $E_{1}$ of $E_{2}$ symmetry species. For the $A$ species $f=4$ if the $K$ of either the upper or the lower states is equal to zero. For each member of a $K$-type doublet, $f=2$ and consequently $f=4$ when the doublet splitting is unobservably small.

In the case of $\mathrm{CD}_{3} \mathrm{OH}$ and $\mathrm{CD}_{3} \mathrm{OD}, f=16$ for states with the symmetry species $E_{1}$ or $E_{2}$. For the states of $A$ species $f=22$ if the $K$ of either the initial or final states is equal to zero. For each member of a $K$-type doublet $f=11$ and thus $f=22$ when the doublet spacing is unobservably small. Incidentally the proportionality constant $A$ is different for each isotopic molecule but this causes no difficulty since we are only interested in the relative intensities of the lines in each spectrum.

$(i|M| j)^{2}$ denotes the sum of the squares of the matrix elcments of the appropriate components of the permanent electric dipole of the molecule. In the present investigation we are concerned with the so called $b$-type transitions in which $\Delta K= \pm 1$ and the component in question is the one which is perpendicular to the methyl symmetry $\mathrm{axis}^{3}$. One of the consequences of the fact that $H^{0}=H_{R s y m}^{0}+H_{T}{ }^{0}$ is that $(i|M| j)^{2}$ can be written as the product of two factors, $S_{R}$, which expresses the intensity of the lines of a rigid symmetrical rotator and $S_{T}$, which arises from the hindered torsional motion. $S_{R}$ for the lines of the $R, Q$ and $P$ branches is given, respectively, by the formulas

$$
\begin{aligned}
\left(J K\left|S_{R}\right| J+1, K \pm 1\right) & =(J \pm K+1)(J \pm K+2) / 2(J+1) \\
\left(J K\left|S_{R}\right| J, K \pm 1\right) & \\
& =(2 J+1)(J \pm K)(J \pm K+1) / 2 J(J+1) \\
\left(J K\left|S_{R}\right| J-1, K\right. & \pm 1) \\
& =(J \mp K)(J \mp K-1) / 2 J .
\end{aligned}
$$

$S_{T}$ depends upon the coefficients $a_{n+\pi m}$ used to describe the torsional part of the wavefunction. The formula in question is

$$
\left(n \tau K\left|S_{T}\right| n^{\prime} \tau^{\prime} K \pm 1\right)=\sum_{m} a_{n \tau K m} a_{n^{\prime} \tau^{\prime} K \pm 1, m \pm 1} .
$$

${ }^{3}$ It proves convenient to absorb the square of the dipole component $\mu_{\perp}{ }^{2}$ into the proportionality factor $A$. 
$n$ and $n^{\prime}$ can have any values but $\tau$ and $\tau^{\prime}$ must be chosen so that both states belong to the same symmetry species.

\section{ANALYSIS OF THE TORSION-ROTATION SPECTRUM OF $\mathrm{CH}_{3} \mathrm{OH}$}

The analysis of the spectrum of $\mathrm{CH}_{3} \mathrm{OH}$ was accomplished through the use of a series of stepwise approximations. The first of these consisted in calculating the energy levels and the wavefunctions (i.e., the $a_{n \tau K m}$ ) for values of $n=0$ to 4, $K=0$ to $20, J=0$ to 40 , and $\tau=1,2$, and 3 . In this initial calculation the inertial constants, the barrier height and the Kirtman perturbation constants were taken from the paper of Lees and Baker using their Fit II.

The frequencies allowed by the selection rules were calculated together with their intensities. Many thousands of lines would result from this process but only those lines were selected which satisfied the criteria that (a) they lay within the region of experimental measurement, which for $\mathrm{CH}_{3} \mathrm{OH}$ extended from $520 \mathrm{~cm}^{-1}$ to $910 \mathrm{~cm}^{-1}$. The second criterion (b) was that no lines with intensities less than $0.6 \%$ of the intensity of the strongest lines were accepted. These selected lines were then plotted as points on the same frequency scale as that used for the observed spectrum. The ordinate of each point was taken equal to the calculated intensity using a convenient scale.

In comparing the calculated and observed spectra, attention was initially directed towards identifying the more intense $Q$ branches. This was often difficult since for $\mathrm{CH}_{3} \mathrm{OH}$ many of the $Q$ branches tend to occur in overlapping groups. However, in a considerable number of cases isolated $Q$ branches could be found and identified. The differences between the observed and calculated positions of these $Q$ branches were usually of the order of one or two waves per centimeter thus indicating that an improved fit might be obtained by altering the values of the constants given by Lees and Baker.

The majority of the $Q$ branches are degraded towards lower frequencies. An example of this is shown in Fig. 1 where (a) and (b) depict the calculated and observed spectra, respectively, for the transition $(323 J) \leftarrow(014 J)$. It will be noted that superimposed upon the $Q$ branch are a number of lines, many of which have been identified as $R$ branch lines belonging to different $n_{\tau} K$ transitions. To the approximation to which we are working the frequency of a $Q$ branch line may be expressed as $\nu_{Q}=q_{0}+q_{1}\left(J^{2}+J\right)+q_{2}\left(J^{2}+J\right)^{2}$ where the smallest value of $J$ is equal to the largest $K$ associated with the transition. Thus in Fig. 1 the first line has $J=4$. The values of $q_{0}, q_{1}$ and $q_{2}$ follow from the energy expression given earlier. It will be seen that $q_{1}$ and $q_{2}$ depend upon the Kirtman constants $F_{v}, G_{v}, L_{v}, D_{J K}$ and upon the asymmetry terms $D_{n \tau K J}$ and $S_{n \tau K J}$. It is just this set of Kirtman constants as well as the asymmetry terms which are most accurately determined by the microwave spectrum and consequently we have used the I,ees and Baker values in calculating $q_{1}$ and $q_{2}$. For the transition shown in Fig. 1 both $q_{1}$ and $q_{2}$ are negative numbers and consequently the $Q$ 


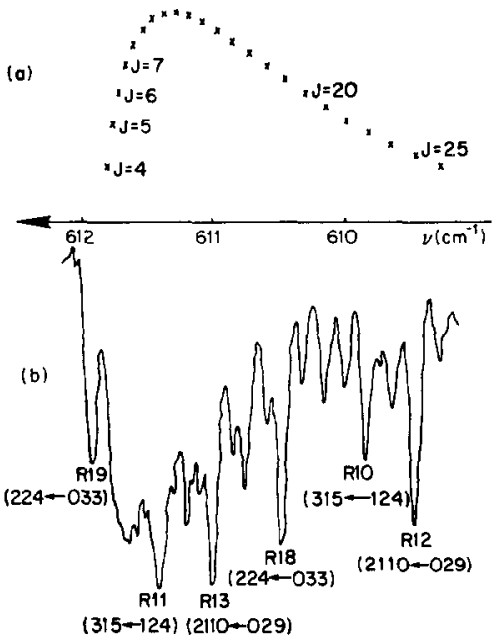

FiG. 1. Calculated and observed $Q$ branch of $\mathrm{CH}_{3} \mathrm{OH}$ for the transition $(323 J) \leftarrow(014 J)$.

(a)

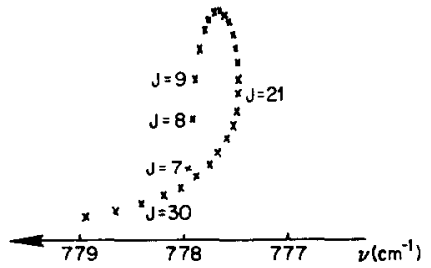

(b)

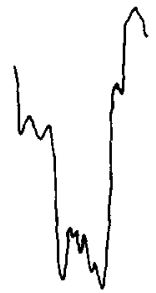

FIG. 2. Calculated and observed $Q$ branch of $\mathrm{CH}_{3} \mathrm{OH}$ for the transition $(337 J) \leftarrow(116 J)$.

branch degrades towards smaller wavenumbers. Clearly it is not possible from the observed spectrum to fix $q_{0}$ with an accuracy greater than about $0.3 \mathrm{~cm}^{-1}$.

A $Q$ branch which is particularly interesting is shown in Fig. 2 and is for the transition $(337 J) \leftarrow(116 J)$. The calculated value of $q_{1}$ is negative while $q_{2}$ is positive. This results in a $Q$ branch which turns back on itself and gives rise to a doublet in the observed spcctrum since the individual lines are not resolved. In this case it is possible to determine $q_{0}$ with somewhat greater accuracy. 
A far more accurate way of determining $q_{0}$ (the $Q$ branch origin) occurs whenever it is possible to identify individual $P$ or $R$ branch lines. These are often the strongest lines in the spectrum. A study of the intensity formula shows that, except for the lowest values of $K$, the $R$ branch lines of a transition where $K+1 \leftarrow$ $K$ will dominate whereas the strongest lines for a transition $K-1 \leftarrow K$ will be those of the $P$ branch. The formulas for the frequencies of the $P$ and $R$ branch lines are readily obtained from the energy and maybe expressed as quartic polynomials in the quantum number $J$. The constant term is $q_{0}$ while the coefficients of the various powers of $J$ are functions of the Kirtman constants $F_{v}, G_{v}, L_{v}, D_{J K}$ and $D_{J J}$ together with the asymmetry terms. In addition to these the coefficient of the linear term contains the quantity $(B+C)$ which is also well determined from the microwave spectrum. $(B+C)$ is the large term which gives the magnitude of the spacing of the lines and for $\mathrm{CH}_{3} \mathrm{OH}$ is approximately equal to $1.61 \mathrm{~cm}^{-1}$. The method for finding the $Q$ origins is now clear. Whenever a series of $P$ or of $R$ branch lines can be identified, $q_{0}$ is cqual to the observed frequency of a particular line minus the contribution due to the remaining portion of the polynomial in $J$. Typically we are able to identify 5-15 $P$ or $R$ branch lines belonging to a particular $n \tau K$ transition and thus obtain that number of independent measurements of $q_{0}$. It is found that these agree among themselves to the order of the experimental error, namely, $0.03 \mathrm{~cm}^{-1}$. Incidentally this agreement constitutes an independant test of the values of the Kirtman constants in question as deduced from the microwave spectrum.

The expression for the $q_{0}$ of the transition $\left(n^{\prime} \tau^{\prime} K^{\prime}\right) \leftarrow(n \tau K)$ follows from the energy formula and may be written

$$
\begin{aligned}
\nu=\left(E_{n^{\prime} \tau^{\prime} K^{\prime}}-\right. & \left.E_{n \tau K}\right) / h c \quad \text { where, } \\
\left(E_{n \pi \mathrm{K}}\right) / h c & =F\left\langle P_{\gamma}{ }^{2}\right\rangle+\frac{1}{2} V_{3}\langle 1-\cos 3 \gamma\rangle+\left[A-\frac{1}{2}(B+C)\right] K^{2} \\
& +\frac{1}{2} V_{6}\langle 1-\cos 6 \gamma\rangle-D_{K K} K^{4}+k_{1} K^{3}\left\langle P_{\gamma}\right\rangle+k_{2} K^{2}\left\langle P_{\gamma}{ }^{2}\right\rangle \\
& +k_{3} K\left\langle P_{\gamma}{ }^{3}\right\rangle+k_{4}\left\langle P_{\gamma}{ }^{4}\right\rangle+k_{5} K^{2}\langle 1-\cos 3 \gamma\rangle \\
& +k_{6} K\left\langle P_{\gamma}(1-\cos 3 \gamma)\right\rangle+k_{7}\left\langle P_{\gamma}{ }^{2}(1-\cos 3 \gamma)\right\rangle+d_{0} .
\end{aligned}
$$

The expectation values are of course evaluated using the basic wavefunctions of $H_{T}{ }^{0}$ for the state $n \tau K$. The last term $d_{0}$ arises from the asymmetry of the molecule. It turns out that $d_{0}$ is smaller than the experimental error for the infra data but it has been included in the microwave $Q$ origins. In the above expression $(B+C)$ has been well determined by Lees and Baker from the microwave spectrum. The constants $F$ and $A$ depend primarily upon the moments of inertia $I_{a 1}$ and $I_{a 2}$ and to a slight extent upon $I_{b}, I_{c}$ and $I_{a b}$.These latter are also accurately known from the microwave spectrum. The frequency of a $Q$ branch origin 
thus depends upon the large constants $I_{a 1}, I_{a 2}$, and $V_{3}$ and upon the perturbation constants $V_{6}, D_{K K}$ and $k_{1}, k_{2}, \cdots, k_{7}$. As stated earlier, the redundancy relation shows that an infinite set of related values for $V_{6}, k_{6}$ and $k_{7}$ together with small changes in the large constants will equally well reproduce the observed spectrum. Wo shall follow the Fit II of Lees and Baker where $k_{7}$ is arbitrarily set equal to zero. It should be emphasized again that there is no physical basis for this choice but also that it will not affect the accuracy of calculating the spectrum.

The initial calculation in which the Lees and Baker constants were used resulted in spectral lines whose positions differed from the observed lines by up to one or two waves per centimeter. It was sufficiently good, however, to allow a unique identification of $13 Q$ branch origins. These were combined with the $16 Q$ branch origins obtained by Jees and Baker from their microwave spectrum. A nonlinear least squares calculation was performed with these 29 data to find the 11 molecular constants $I_{a 1}, \cdots, k_{6}$. It was found that 3 or 4 iterations sufficed to yicld convergent values for the constants. The spectrum was now recomputed and further identifications were looked for and found in the observed spectrum. By repeating this process it has been possible to identify 39 infrared $Q$ branch origins with an accuracy which we estimate to be $0.03 \mathrm{~cm}^{-1}$. These together with the 16 microwave $Q$ origins constitute the 55 data with which to determine the 11 molecular parameters. A nonlinear least squares solution yielded the values appearing in Table I. The least squares treatment takes account of the accuracy of measurement of the $Q$ origins which, for the microwave data, has been taken from the paper by Lees and Baker.

The agreement between the perturbation constants we find and those listed by Lees and Baker appears to be only moderately good and in most cases lies well outside the probable errors resulting from the least squares solutions. It seems likely to us that the reason lies in the nature of a least squares treatmont which rests upon the assumption that all errors arise from accidental sources and that there are no systematic errors. In our case this assumption fails in two respects. The $Q$ branch origins used by Lees and Baker came from the center of gravity of the $Q$ branch lines as observed by Burkhard and Dennison (8). These differ from the true origins by amounts of the order of $0.5 \mathrm{~cm}^{-1}$ and the errors so introduced are to a great extent systematic rather than purely accidental. From the theoretical side there are also what may be called systematic errors. The theoretical formula is a development which includes only the first order terms arising from the nonrigid nature of the molecule. The magnitude of these perturbation terms for the infrared $Q$ origins in question is often around $10 \mathrm{~cm}^{-1}$ or even larger. It is to be expected that higher order perturbations will contribute corrections which may be substantially greater than the experimental errors. It is for this reason that we do not believe that any of the probable errors appearing in Table I are to be taken too seriously although they do indicate in some measure the accuracy of the fit. 


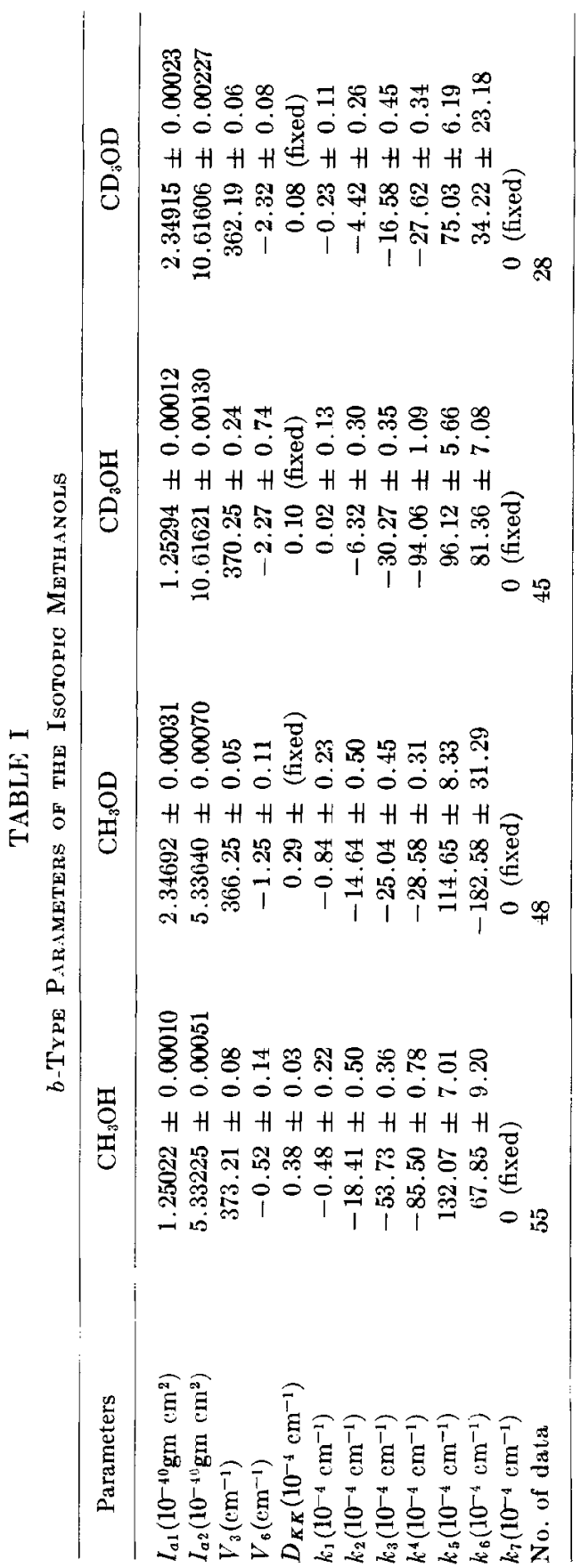


Table II lists the data from the observed and computed spectra. The first column gives the transition and the second column the observed $Q$ branch origins where the first 16 entries are from the work of Lees and Baker. The third column contains the estimates of the experimental accuracy which were used in the least squares solution. The fourth column gives $\Delta_{1}=\nu_{\text {obsd }}-\nu_{\text {caled }}$. The fifth column $\Delta_{2}$ will be discussed in the last section of our paper.

It will be noted that the agreement is generally very good even though the majority of the $\Delta_{1}$ are somewhat larger than the experimental accuracies. Two reasons suggest themselves for these deviations: (1) It is possible that our estimate of the experimental accuracy is too low, (2) The infrared transitions in question involve high states of torsion and of $K$. It is just these states which would be most affected by the neglect of higher order perturbation terms.

As a final test it was decided to attempt to reconstruct by computation several portions of the spectrum and compare these with the spectrum observed by Woods and Peters. One such portion is shown in Fig. 3. In the upper part, labeled Absorption Coefficient, the computed lines are plotted as points whose ordinates are proportional to the calculated intensities. Many of the points are labeled with their transitions but other points are unlabeled to avoid confusion. The computer plotted alternate lines in frequency with the symbols $X$ and $\|$. It is quite easy to recognize the group of $Q$ branches in the region near 780 $\mathrm{cm}^{-1}$ as well as some of the stronger $P$ and $R$ branch series of lines.

In the upper part of Fig. 3 there is also plotted the absorption coefficient as it would be observed by a spectrometer of infinitely high resolving power. The calculation of this curve requires a knowledge of the shape of the spectral lines. Woods and Peters used a pressure of 40 Torr in mapping this portion of the spectrum. It is therefore expected that the lines will conform to a Lorentzian shape, namely, $\left.\alpha(v)=\gamma I / \pi\left[v-\nu_{0}\right)^{2}+\gamma^{2}\right]$ where $2 \gamma$ is the half width and $I$ is the intensity of the line. Woods and Peters estimate that for a pressure of 40 Torr $\gamma=0.018 \mathrm{~cm}^{-1}$ but by a process of trial and error we find that a somewhat better fit with experiment may be made by choosing $\gamma=0.026 \mathrm{~cm}{ }^{1}$. 'The resulting absorption coefficient $\alpha(\nu)$ is found by summing the contributions from all lines in the neighborhood of $\nu$.

Finally, the computation of the transmission at the frequency $\nu^{\prime}$ requires the spectrometer profile function $\rho\left(v^{\prime}, v\right)$ as determined experimentally from the central image:

$$
T\left(\nu^{\prime}\right)=\int_{0}^{\infty} \rho\left(\nu^{\prime}, \nu\right) e^{-c \alpha} d \nu / \int_{0}^{\infty} \rho d \nu .
$$

The proportionality constant $C$ which contains the pressure and the path length is chosen so as to give the best fit with experiment. The result of this lengthy calculation is shown in the middle portion of Fig. 3. The agreement between this curve and the observed spectrum is striking. A careful examination of Fig. 
TABLE II

Observed and Calculated $Q$ Branch Origins of $\mathrm{CH}_{3} \mathrm{OH}$

\begin{tabular}{|c|c|c|c|c|}
\hline$\left(\mathrm{n}^{\prime} \boldsymbol{\tau}^{\prime} \mathbf{K}^{\prime}\right) \leftarrow\left(\mathrm{n}_{\tau} \mathbf{K}\right)$ & $\nu_{\text {obsd }}\left(\mathrm{cm}^{-1}\right)^{a}$ & Acc. $\left(\mathrm{cm}^{-1}\right)$ & $\Delta_{1}\left(\mathrm{~cm}^{-1}\right)$ & $\Delta_{\mathrm{q}}\left(\mathrm{cm}^{-1}\right)$ \\
\hline$\left(\begin{array}{ll}02 & 1\end{array}\right) \leftarrow\left(\begin{array}{ll}03 & 0\end{array}\right)$ & 5.505432 & 0.000008 & -0.000013 & 0.000029 \\
\hline$(012) \leftarrow(021)$ & 0.832264 & 0.000008 & 0.000014 & -0.000043 \\
\hline$(015) \leftarrow(024)$ & 27.8559 & 0.0022 & -0.0014 & 0.0009 \\
\hline$(036) \leftarrow(015)$ & 29.7105 & 0.0033 & -0.0004 & 0.0015 \\
\hline$(020) \leftarrow\left(\begin{array}{ll}01 & 1\end{array}\right)$ & 5.245801 & 0.000008 & 0.000018 & -0.000067 \\
\hline$(03 \quad 2) \leftarrow(01 \quad 1)$ & 14.124150 & 0.000033 & -0.000025 & 0.000113 \\
\hline$(014) \leftarrow(023)$ & 17.51339 & 0.00050 & 0.00002 & 0.00082 \\
\hline$(035) \leftarrow(014)$ & 25.10113 & 0.00060 & -0.00113 & -0.00063 \\
\hline$(031) \leftarrow(010)$ & 10.105220 & 0.000033 & 0.000029 & -0.000033 \\
\hline$(022) \leftarrow\left(\begin{array}{lll}03 & 1\end{array}\right)$ & 16.10266 & 0.00017 & -0.00014 & -0.00016 \\
\hline$(013) \leftarrow(022)$ & 8.404470 & 0.000017 & -0.000005 & 0.000003 \\
\hline$\left(\begin{array}{lll}03 & 4\end{array}\right) \leftarrow\left(\begin{array}{ll}01 & 3\end{array}\right)$ & 21.23096 & 0.00067 & 0.00002 & 0.00035 \\
\hline$(025) \leftarrow(034)$ & 40.0043 & 0.0100 & 0.0006 & -0.0041 \\
\hline$\left(\begin{array}{ll}01 & 6\end{array}\right) \leftarrow(025)$ & 38.7984 & 0.0067 & -0.00018 & 0.0034 \\
\hline$(130) \leftarrow\left(\begin{array}{ll}12 & 1\end{array}\right)$ & 6.327312 & 0.000017 & 0.000036 & 0.000045 \\
\hline$\left(\begin{array}{ll}13 & 1\end{array}\right) \leftarrow\left(\begin{array}{ll}12 & 2\end{array}\right)$ & 18.47610 & 0.00017 & -0.00117 & 0.00057 \\
\hline$(313) \leftarrow\left(\begin{array}{ll}12 & 2\end{array}\right)$ & 692.34 & 0.03 & -0.05 & 0.21 \\
\hline$(333) \leftarrow(124)$ & 693.78 & 0.03 & 0.00 & 0.11 \\
\hline$\left(\begin{array}{ll}32 & 1\end{array}\right) \leftarrow\left(\begin{array}{ll}03 & 0\end{array}\right)$ & 694.70 & 0.03 & -0.00 & -0.23 \\
\hline$(317) \leftarrow(026)$ & 697.89 & 0.03 & -0.10 & -0.13 \\
\hline$(338) \leftarrow\left(\begin{array}{ll}11 & 7\end{array}\right)$ & 733.70 & 0.03 & 0.17 & 0.67 \\
\hline$(330) \leftarrow(021)$ & 736.44 & 0.03 & 0.04 & -0.17 \\
\hline$(334) \leftarrow(125)$ & 736.90 & 0.03 & -0.05 & 0.18 \\
\hline$(312) \leftarrow(121)$ & 737.43 & 0.03 & -0.06 & 0.23 \\
\hline$(316) \leftarrow(025)$ & 737.54 & 0.03 & -0.09 & -0.08 \\
\hline$(337) \leftarrow(116)$ & 778.09 & 0.03 & 0.13 & 0.73 \\
\hline$(3311) \leftarrow(0110)$ & 778.74 & 0.03 & 0.10 & 0.20 \\
\hline$(315) \leftarrow(024)$ & 779.87 & 0.03 & -0.07 & -0.04 \\
\hline$(311) \leftarrow(120)$ & 780.07 & 0.03 & -0.11 & 0.21 \\
\hline$(331) \leftarrow(022)$ & 781.05 & 0.03 & 0.05 & -0.13 \\
\hline$(336) \leftarrow(115)$ & 820.19 & 0.03 & 0.06 & 0.73 \\
\hline$(3310) \leftarrow(019)$ & 821.58 & 0.03 & 0.09 & 0.31 \\
\hline$(314) \leftarrow(023)$ & 825.21 & 0.03 & -0.05 & 0.00 \\
\hline$\left(\begin{array}{ll}33 & 2\end{array}\right) \leftarrow(023)$ & 828.76 & 0.03 & 0.02 & -0.10 \\
\hline$(313) \leftarrow(02 \quad 2)$ & 873.71 & 0.03 & -0.07 & -0.01 \\
\hline$(226) \leftarrow(035)$ & 516.99 & 0.03 & -0.10 & -0.35 \\
\hline$(2112) \leftarrow(0211)$ & 527.79 & 0.03 & -0.12 & -0.54 \\
\hline$\left(\begin{array}{ll}22 & 1\end{array}\right) \leftarrow(012)$ & 539.84 & 0.03 & 0.07 & -0.27 \\
\hline$(31 \quad 6) \leftarrow\left(\begin{array}{ll}12 & 5\end{array}\right)$ & 541.06 & 0.03 & -0.08 & 0.07 \\
\hline$(330) \leftarrow\left(\begin{array}{ll}12 & 1\end{array}\right)$ & 548.45 & 0.03 & 0.00 & 0.01 \\
\hline$(225) \leftarrow(034)$ & 549.15 & 0.03 & -0.07 & -0.30 \\
\hline$(222) \leftarrow(013)$ & 574.70 & 0.03 & 0.07 & -0.23 \\
\hline$\left(\begin{array}{ll}22 & 4\end{array}\right) \leftarrow\left(\begin{array}{ll}03 & 3\end{array}\right)$ & 582.82 & 0.03 & -0.08 & -0.30 \\
\hline$(2110) \leftarrow(029)$ & 590.68 & 0.03 & -0.15 & -0.34 \\
\hline
\end{tabular}

a The 16 microwave transitions were observed by Lees and Baker (1). 
TABLE II-Continued

\begin{tabular}{|c|c|c|c|c|}
\hline$\left(\mathrm{n}^{\prime} \tau^{\prime} \mathbf{K}^{\prime}\right) \leftarrow(\mathrm{n} \tau \mathbf{K})$ & $\nu_{\text {obsd }}\left(\mathrm{cm}^{-1}\right)$ & Acc. $\left(\mathrm{cm}^{-1}\right)$ & $\Delta_{1}\left(\mathrm{~cm}^{-1}\right)$ & $\Delta_{Z}\left(\mathrm{~cm}^{-1}\right)$ \\
\hline$(315) \leftarrow(124)$ & 594.00 & 0.03 & -0.08 & 0.12 \\
\hline$\left(\begin{array}{ll}33 & 1\end{array}\right) \leftarrow\left(\begin{array}{ll}12 & 2\end{array}\right)$ & 599.64 & 0.03 & 0.02 & 0.05 \\
\hline$(323), \quad(014)$ & 611.89 & 0.03 & 0.10 & 0.18 \\
\hline$(223) \leftarrow\left(\begin{array}{ll}2 & 3\end{array}\right)$ & 617.94 & 0.03 & -0.07 & -0.28 \\
\hline$(219) \leftarrow(028)$ & 624.55 & 0.03 & -0.14 & -0.25 \\
\hline$(314) \leftarrow\left(\begin{array}{ll}12 & 3\end{array}\right)$ & 644.52 & 0.03 & -0.07 & 0.16 \\
\hline$\left(\begin{array}{ll}33 & 2\end{array}\right) \leftarrow\left(\begin{array}{ll}12 & 3\end{array}\right)$ & 648.09 & 0.03 & 0.01 & 0.08 \\
\hline$(324) \leftarrow(015)$ & 650.35 & 0.03 & 0.08 & -0.12 \\
\hline$(322) \leftarrow(031)$ & 655.58 & 0.03 & -0.01 & -0.25 \\
\hline$(339) \leftarrow\left(\begin{array}{ll}11 & 8\end{array}\right)$ & 686.69 & 0.03 & 0.18 & 0.55 \\
\hline$(325) \leftarrow\left(\begin{array}{ll}01 & 6\end{array}\right)$ & 691.37 & 0.03 & 0.07 & -0.01 \\
\hline
\end{tabular}

3 on a larger scale shows that many of the computed transmission peaks are not due to single lines but rather to the chance piling up of faint lines. Since our calculation uses a cut-off for the weakest lines this may account for some of the differences between the computed and observed spectra.

\section{ANALYSIS OF THE TORSION-ROTATION SPECTRA OF $\mathrm{CH}_{3} \mathrm{OD}, \mathrm{CD}_{3} \mathrm{OH}$ AND $\mathrm{CD}_{3} \mathrm{OD}$}

The analyses of the spectra of the isotopic methanols $\mathrm{CH}_{3} \mathrm{OD}$ and $\mathrm{CD}_{3} \mathrm{OII}$ followed the same pattern as that used for $\mathrm{CH}_{3} \mathrm{OH}$. Since no high resolution infrared rotation spectra had been measured at the time of their analysis, Iees and Baker were forced to rely entirely upon their observed microwave spectra. These served to determine with accuracy the constants which multiply the various powers of $\left(J^{2}+J\right)$, namely, $(B+C), F_{v}, G_{v}, I_{v}, D_{J J}, D_{J K}, D_{a b}$ and $(B-C)$. In addition to these they evaluated $I_{a 1}, I_{a 2}$ and $V_{3}$ but here the accuracy was not as high since the remaining Kirtman constants were necessarily set equal to zero.

Using the values of the above paramcters as listed by Lees and Baker we calculated the positions and intensities of the lines falling within the spectral regions observed by Woods and Peters. The identification of the $Q$ origins was somewhat more difficult since the calculated positions differed from the actual positions by amounts of the order of $5-10 \mathrm{em}^{-1}$. These larger differences occurred since so many of the important Kirtman constants had been neglected in this initial calculation. However, certain identifications could be made and hence a new computed spectrum could be obtained. By repeating this process several times it was possible to fix the frequencies of 45 infrared $Q$ origins for $\mathrm{CH}_{3} \mathrm{OD}$ and 40 origins for $\mathrm{CD}_{3} \mathrm{OH}$. The accuracy for most of these was $0.03 \mathrm{~cm}^{-1}$. The full set of Kirtman parameters was now calculated by nonlinear least squares and these are listed in Table I where, as before, Fit II was used. The $D_{K \kappa}$ were 


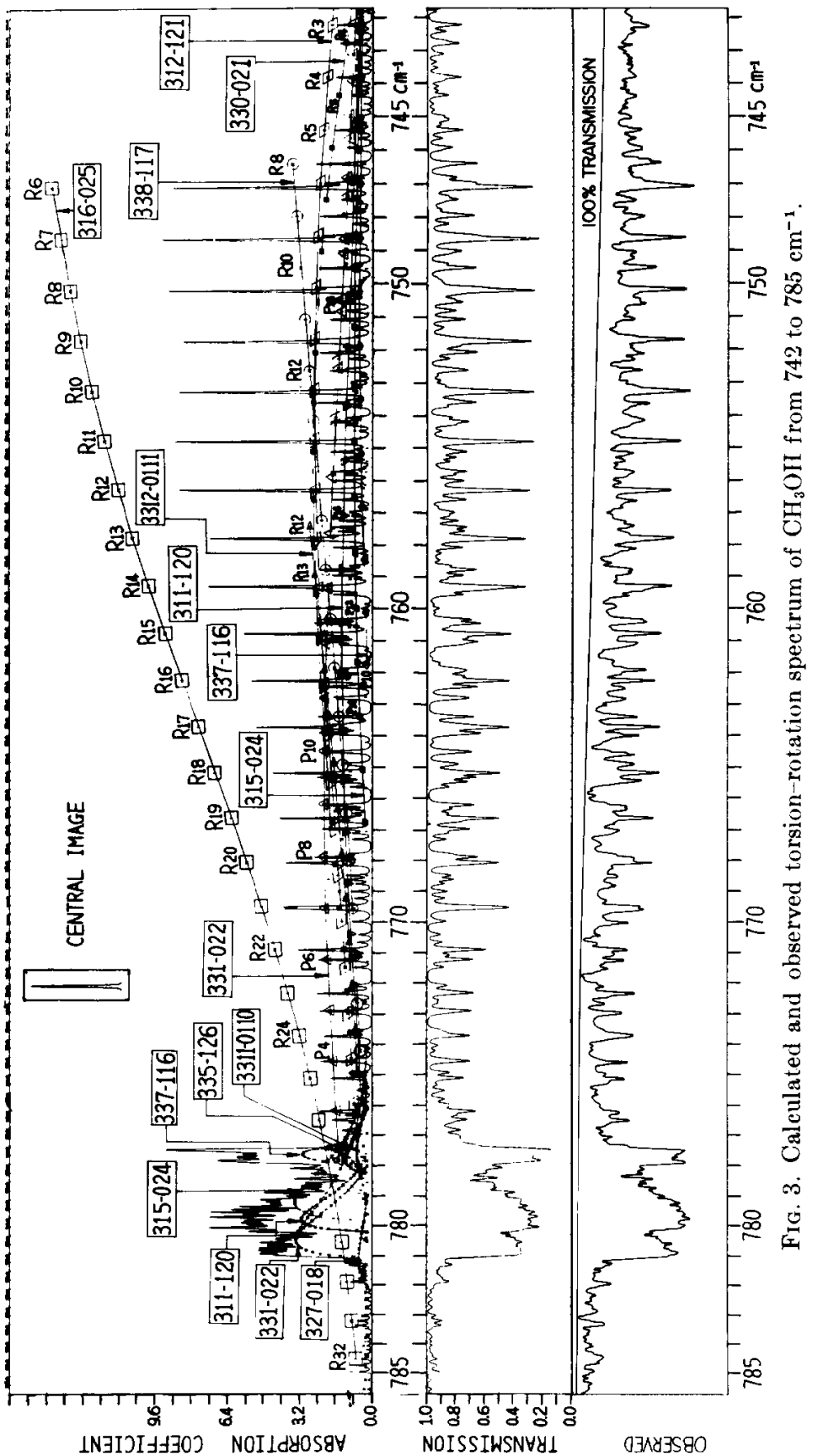


fixed through an auxiliary calculation using the force constants from a normal coordinate analysis ${ }^{4}$. The positions of the $Q$ origins were now computed and compared with the observed positions. The results appear in Tables III and IV. Again the agreement between the observed and computed frequencies is very good.

The situation for the all heavy isotope $\mathrm{CD}_{3} \mathrm{OD}$ differed from the foregoing in the following respects. The measurements of I.ees (2) of the microwave spectrum of this molecule did not include lines from which the perturbation constants $L_{x}, D_{J J}$ and $D_{J K}$ could be evaluated. We estimated their values by examining the constants of the other isotopic molecules and attempting an ompirical extrapolation. Following the type of reasoning to be presented in the next section it was inferred that $D_{J J} /(B+C)^{2}$ should be approximately constant for all the isotopic molecules. This conjecture is quite well satisfied for the first three isotopic molecules and leads to the estimate $D_{J J}=0.009 \times 10^{-4}$ $\mathrm{cm}^{-1}$ for $\mathrm{CD}_{3} \mathrm{OD}$. In a similar way it was thought that $D_{J K} / A(B+C)$ might be constant and this again was found to be approximately true for the three molecules where the data are known. The resulting value for $\mathrm{CD}_{3} \mathrm{OD}$ was $D_{J K}=$ $0.040 \times 10^{-4} \mathrm{~cm}^{-1}$. An estimate of $L_{v}$ was made in the following way. Sixtren of the longest and best identified $P$ and $R$ branch series were selected. The spacing of the lines in any one series are linear functions of $1 / 2(B+C), F_{v}, C_{v}$ and the asymmetry terms. These are all well determined from Lees' microwave spectrum. The spacings are also functions of $D_{J J}$ and $D_{J K}$ for which the above estimated values were used and of $L_{t}$. A least squares solution was made with

${ }^{4}$ The Kirtman constants as initially found by the least squares fit appeared to have reasonable values with one exception. The $D_{K K}$ was poorly determined. This probably arose from the fact that the contributions from the perturbation term in question were generally very small. An auxilliary method of finding $D_{K K}$ was looked for. The normal vibration problem of the isotopic methanols has been treated by M. Margottin-Maclou (11). In addition to finding the potential constants she calculated $D_{K K}, D_{J K}$ and $D_{J J}$ for $\mathrm{CH}_{3} \mathrm{OH}$. Her values, $0.39,0.107$ and $0.018 \times 10^{-4} \mathrm{~cm}^{-1}$ are in good agreement with the experimental values of Lees and Baker, namely, $0.42,0.096$ and $0.016 \times 10^{-4}$. (The value of $D_{K K}$ we obtained was $0.38 \times 10^{-4}$ )

Since the $D_{f K}$ and $D_{f J}$ appear to be fixed with some aceuracy for $\mathrm{CH}_{3} \mathrm{OD}$ and $\mathrm{Cl}_{3} \mathrm{OH}$ by the experiments of Lees and Baker we elected to make the relatively simple calculation of $D_{s}=D_{K K}+D_{J K}+D_{J J}$ and obtain the $D_{K K}$ by subtraction. For a symmetrical molecule $D_{s}$ may be derived by considering the rotations of the molecule about its symmetry axis where $J=K$. Standard perturbation methods ( $(7)$ lead to the formula

$$
D_{s}=\frac{h^{4}}{8 I_{a}{ }^{4}} \sum_{i} \frac{C_{i}^{2}}{k_{i}}
$$

where $k_{i}$ is the force constant for the $i$-th normal vibration. $C_{i}=\left(\delta I a / \delta q_{i}\right)_{0}$ is the derivative of the moment of inertia with respect to the $i$-th normal coordinate. Using the potential constants of Margottin-Maclou we repeated her normal coordinate analysis and found the values $0.505,0.369,0.164$ and $0.129 \times 10^{-4} \mathrm{~cm}^{-1}$ for $\mathrm{CH}_{3} \mathrm{OH}, \mathrm{CH}_{3} \mathrm{OD}, \mathrm{CD}_{3} \mathrm{OH}$ and $\mathrm{CD}_{3} \mathrm{OD}$, respectively. 
TABLE III

Observed ano Calculated $Q$ Branch Origins of $\mathrm{CH}_{3} \mathrm{O}$ )

\begin{tabular}{|c|c|c|c|c|c|}
\hline$\left(\mathrm{n}^{\prime} \tau^{\prime} \mathbf{K}^{\prime}\right)$ & $\leftarrow(\mathrm{n} \tau \mathrm{K})$ & $\nu_{\mathrm{obad}}\left(\mathrm{cm}^{-1}\right)$ & Acc. $\left(\mathrm{cm}^{-1}\right)$ & $\Delta_{1}\left(\mathrm{~cm}^{-1}\right)$ & $\Delta_{2}\left(\mathrm{~cm}^{-1}\right)$ \\
\hline$\left(\begin{array}{ll}02 & 1\end{array}\right)$ & $\leftarrow\left(\begin{array}{ll}03 & 0\end{array}\right)$ & $3.674467^{\mathrm{a}}$ & 0.000033 & -0.0000780 & -0.000063 \\
\hline$(012)$ & $\leftarrow(021)$ & $6.559478^{\mathrm{a}}$ & 0.000033 & 0.0000525 & 0.000058 \\
\hline$\left(\begin{array}{ll}01 & 1\end{array}\right)$ & $\leftarrow(020)$ & $0.631205^{\mathrm{a}}$ & 0.000033 & -0.0000827 & -0.000202 \\
\hline$(217)$ & $\leftarrow(026)$ & 395.49 & 0.03 & 0.07 & 0.33 \\
\hline$(223)$ & $\leftarrow(032)$ & 398.04 & 0.03 & 0.05 & 0.40 \\
\hline$(3210)$ & $\leftarrow(139)$ & 394.51 & 0.03 & -0.01 & 0.17 \\
\hline$(336)$ & $\leftarrow(11.5)$ & 410.49 & 0.03 & 0.00 & 0.24 \\
\hline$\left(\begin{array}{ll}31 & 2\end{array}\right)$ & $\leftarrow(121)$ & 424.79 & 0.03 & 0.06 & 0.21 \\
\hline$(2310)$ & $\leftarrow(019)$ & 423.34 & 0.03 & 0.04 & 0.03 \\
\hline$\left(\begin{array}{ll}21 & 6\end{array}\right)$ & $\leftarrow(025)$ & 427.02 & 0.03 & 0.00 & 0.32 \\
\hline$\left(\begin{array}{ll}32 & 2\end{array}\right)$ & $\leftarrow\left(\begin{array}{ll}01 & 3\end{array}\right)$ & 441.72 & 0.05 & -0.04 & 0.10 \\
\hline$\left(\begin{array}{ll}31 & 6\end{array}\right)$ & $\leftarrow(037)$ & 449.26 & 0.03 & -0.08 & -0.37 \\
\hline$\left(\begin{array}{ll}32 & 9\end{array}\right)$ & $\leftarrow(138)$ & 441.80 & 0.03 & -0.07 & 0.05 \\
\hline$(23$ 9 $)$ & $\leftarrow\left(\begin{array}{ll}018 & 8\end{array}\right)$ & 455.77 & 0.03 & -0.02 & 0.06 \\
\hline$\left(\begin{array}{ll}33 & 5\end{array}\right)$ & $\leftarrow(114)$ & 456.56 & 0.03 & -0.03 & 0.13 \\
\hline$\left(\begin{array}{ll}31 & 1\end{array}\right)$ & $\leftarrow(120)$ & 469.52 & 0.03 & -0.01 & 0.08 \\
\hline$\left(\begin{array}{ll}31 & 5\end{array}\right)$ & $\leftarrow(024)$ & 472.69 & 0.03 & 0.16 & 0.31 \\
\hline$\left(\begin{array}{ll}32 & 1\end{array}\right)$ & $\leftarrow(030)$ & 472.59 & 0.03 & 0.07 & 0.38 \\
\hline$(333)$ & $\leftarrow(124)$ & 481.01 & 0.03 & 0.02 & 0.02 \\
\hline$(328)$ & $\leftarrow(137)$ & 487.18 & 0.03 & -0.05 & -0.02 \\
\hline$\left(\begin{array}{ll}33 & 8\end{array}\right)$ & $\leftarrow(017)$ & 495.58 & 0.03 & 0.13 & 0.35 \\
\hline$\left(\begin{array}{ll}33 & 4\end{array}\right)$ & $\leftarrow\left(\begin{array}{ll}11 & 3\end{array}\right)$ & 500.85 & 0.03 & 0.00 & 0.10 \\
\hline$\left(\begin{array}{ll}31 & 4\end{array}\right)$ & $\leftarrow(023)$ & 503.69 & 0.03 & 0.03 & 0.38 \\
\hline$(434)$ & $\leftarrow(125)$ & 524.89 & 0.08 & 0.09 & 0.06 \\
\hline$\left(\begin{array}{ll}32 & 4\end{array}\right)$ & $\leftarrow(015)$ & 526.90 & 0.03 & -0.09 & -0.18 \\
\hline (3111) & $\leftarrow(1210)$ & 516.48 & 0.03 & -0.06 & -0.32 \\
\hline$(327)$ & $\leftarrow(136)$ & 530.99 & 0.03 & -0.05 & -0.08 \\
\hline$\left(\begin{array}{ll}33 & 7\end{array}\right)$ & $\leftarrow\left(\begin{array}{ll}01 & 6\end{array}\right)$ & 534.70 & 0.03 & -0.02 & 0.22 \\
\hline$\left(\begin{array}{ll}43 & 3\end{array}\right)$ & $\leftarrow\left(\begin{array}{ll}11 & 2\end{array}\right)$ & 544.38 & 0.03 & -0.01 & 0.04 \\
\hline$\left(\begin{array}{ll}31 & 3\end{array}\right)$ & $\leftarrow(022)$ & 547.08 & 0.03 & -0.03 & 0.31 \\
\hline$(435)$ & $\leftarrow(126)$ & 572.92 & 0.15 & 0.07 & -0.02 \\
\hline$(3210)$ & $\leftarrow\left(\begin{array}{ll}03 & 9\end{array}\right)$ & 565.41 & 0.03 & -0.04 & -0.13 \\
\hline$\left(\begin{array}{ll}42 & 6\end{array}\right)$ & $\leftarrow(135)$ & 574.76 & 0.03 & -0.02 & -0.10 \\
\hline$\left(\begin{array}{ll}33 & 6\end{array}\right)$ & $\leftarrow(015)$ & 579.42 & 0.03 & -0.04 & 0.23 \\
\hline$\left(\begin{array}{ll}41 & 3\end{array}\right)$ & $\leftarrow(234)$ & 591.05 & 0.03 & -0.09 & -0.29 \\
\hline$\left(\begin{array}{ll}43 & 2\end{array}\right)$ & $\leftarrow\left(\begin{array}{ll}11 & 1\end{array}\right)$ & 590.08 & 0.03 & 0.00 & 0.03 \\
\hline$\left(\begin{array}{ll}31 & 2\end{array}\right)$ & $\leftarrow(021)$ & 594.92 & 0.09 & -0.01 & 0.29 \\
\hline$(4313)$ & $\leftarrow(1112)$ & 587.85 & 0.03 & 0.06 & -0.72 \\
\hline$\left(\begin{array}{ll}42 & 4\end{array}\right)$ & $\leftarrow\left(\begin{array}{ll}23 & 3\end{array}\right)$ & 605.24 & 0.03 & -0.05 & -0.33 \\
\hline$\left(\begin{array}{ll}41 & 9\end{array}\right)$ & $\leftarrow(128)$ & 604.35 & 0.03 & 0.01 & -0.32 \\
\hline$\left(\begin{array}{ll}32 & 9\end{array}\right)$ & $\leftarrow(03.8)$ & 611.31 & 0.03 & -0.07 & -0.08 \\
\hline$\left(\begin{array}{ll}42 & 5\end{array}\right)$ & $\leftarrow(134)$ & 622.40 & 0.03 & 0.00 & -0.06 \\
\hline$\left(\begin{array}{ll}33 & 5\end{array}\right)$ & $\leftarrow\left(\begin{array}{ll}01 & 1\end{array}\right)$ & 628.46 & 0.05 & -0.05 & 0.23 \\
\hline$(414)$ & $\leftarrow(235)$ & 636.27 & 0.03 & 0.11 & 0.00 \\
\hline$\left(\begin{array}{ll}43 & 1\end{array}\right)$ & $\leftarrow\left(\begin{array}{ll}11 & 0\end{array}\right)$ & 645.36 & 0.03 & -0.02 & -0.02 \\
\hline$(417)$ & $\leftarrow(226)$ & 637.51 & 0.03 & 0.20 & -0.32 \\
\hline$\left(\begin{array}{ll}42 & 3\end{array}\right)$ & $\leftarrow\left(\begin{array}{ll}23 & 2\end{array}\right)$ & 654.69 & 0.04 & -0.12 & -0.49 \\
\hline$(328)$ & $\leftarrow\left(\begin{array}{ll}03 & 7\end{array}\right)$ & 661.50 & 0.05 & -0.06 & 0.00 \\
\hline
\end{tabular}

- Observed by Lees and Baker (1). 
TABLE IV

Observed and Calculated $Q$ Branch Origins of $\mathrm{CD}_{3} \mathrm{OH}$

\begin{tabular}{|c|c|c|c|c|}
\hline$\left(n^{\prime} \tau^{\prime} K^{\prime}\right) \leftarrow-(n \boldsymbol{K} K)$ & $\nu_{\text {obsd }}\left(\mathrm{cm}^{-1}\right)$ & Acc. $\left(\mathrm{cm}^{-1}\right)$ & $\Delta_{1}\left(\mathrm{~cm}^{-1}\right)$ & $\Delta_{2}\left(\mathrm{~cm}^{-1}\right)$ \\
\hline$\left(\begin{array}{ll}02 & 1\end{array}\right) \leftarrow\left(\begin{array}{ll}03 & 0\end{array}\right)$ & $2.649197^{\mathrm{a}}$ & 0.000033 & -0.000081 & -0.001507 \\
\hline$\left(\begin{array}{ll}02 & 1\end{array}\right) \leftarrow\left(\begin{array}{ll}01 & 2\end{array}\right)$ & $2.570416^{\mathrm{a}}$ & 0.000033 & 0.000045 & 0.000354 \\
\hline$\left(\begin{array}{ll}02 & 0\end{array}\right) \leftarrow\left(\begin{array}{ll}01 & 1\end{array}\right)$ & $5.362588^{\mathrm{a}}$ & 0.000033 & 0.000035 & 0.000571 \\
\hline$(031) \leftarrow(010)$ & $7.843297^{a}$ & 0.000033 & -0.000013 & 0.000146 \\
\hline$(013) \leftarrow\left(\begin{array}{lll}02 & 2\end{array}\right)$ & $0.577987^{\mathrm{a}}$ & 0.000033 & 0.000054 & 0.000717 \\
\hline$(2210) \leftarrow(039)$ & 492.40 & 0.03 & -0.16 & 0.11 \\
\hline$(222) \leftarrow(013)$ & 504.09 & 0.03 & 0.06 & -0.04 \\
\hline$(229) \leftarrow(038)$ & 508.70 & 0.03 & -0.13 & 0.08 \\
\hline$\left(\begin{array}{ll}33 & 1\end{array}\right) \leftarrow\left(\begin{array}{ll}12 & 2\end{array}\right)$ & 517.53 & 0.03 & 0.07 & -0.16 \\
\hline$\left(\begin{array}{ll}22 & 3\end{array}\right) \leftarrow\left(\begin{array}{ll}01 & 4\end{array}\right)$ & 521.64 & 0.03 & 0.08 & -0.01 \\
\hline$(228) \leftarrow(037)$ & 525.44 & 0.03 & -0.12 & 0.02 \\
\hline$(224) \leftarrow(015)$ & 539.44 & 0.03 & 0.10 & 0.02 \\
\hline$(319) \leftarrow(128)$ & 531.83 & 0.03 & 0.20 & -0.16 \\
\hline$(227) \leftarrow(036)$ & 542.67 & 0.03 & -0.08 & 0.00 \\
\hline$(332) \leftarrow(123)$ & 543.54 & 0.03 & 0.04 & -0.24 \\
\hline$(325) \leftarrow(016)$ & 559.45 & 0.03 & 0.02 & 0.04 \\
\hline$(226) \leftarrow(035)$ & 560.29 & 0.03 & -0.06 & -0.03 \\
\hline$(318),(127)$ & 558.17 & 0.03 & 0.14 & -0.16 \\
\hline$\left(\begin{array}{ll}33 & 3\end{array}\right) \leftarrow(124)$ & 568.90 & 0.03 & 0.03 & -0.29 \\
\hline$(326) \leftarrow(017)$ & 577.80 & 0.03 & 0.06 & 0.09 \\
\hline$(225) \leftarrow(034)$ & 577.78 & 0.03 & 0.01 & -0.06 \\
\hline$(2116) \leftarrow(0215)$ & 580.63 & 0.05 & -0.01 & 0.18 \\
\hline$(317) \leftarrow(126)$ & 583.89 & 0.03 & 0.09 & -0.18 \\
\hline$(334) \leftarrow(125)$ & 593.52 & 0.03 & -0.01 & -0.38 \\
\hline$(327) \leftarrow(018)$ & 597.34 & 0.03 & 0.07 & 0.17 \\
\hline$(324) \leftarrow\left(\begin{array}{lll}03 & 3\end{array}\right)$ & 597.96 & 0.03 & -0.05 & -0.10 \\
\hline$(2115) \leftarrow(0214)$ & 598.22 & 0.05 & 0.02 & 0.18 \\
\hline$(316) \leftarrow(125)$ & 608.92 & 0.03 & 0.02 & -0.24 \\
\hline$(328) \leftarrow(019)$ & 617.51 & 0.03 & 0.03 & 0.22 \\
\hline$(323) \leftarrow(032)$ & 616.98 & 0.03 & -0.02 & -0.12 \\
\hline$(335) \leftarrow(126)$ & 617.30 & 0.03 & -0.17 & -0.60 \\
\hline$(329) \leftarrow(0110)$ & 638.37 & 0.05 & 0.01 & 0.32 \\
\hline$(322) \leftarrow(031)$ & 636.71 & 0.03 & -0.07 & -0.21 \\
\hline$(315) \leftarrow(124)$ & 633.22 & 0.05 & -0.08 & -0.33 \\
\hline$(3113) \leftarrow(0212)$ & 636.01 & 0.03 & 0.00 & 0.17 \\
\hline$(3112) \leftarrow(0211)$ & 655.26 & 0.03 & 0.00 & 0.14 \\
\hline$(314) \leftarrow(123)$ & 656.77 & 0.03 & 0.23 & -0.50 \\
\hline$(337) \leftarrow(128)$ & 663.08 & 0.08 & -0.23 & -0.73 \\
\hline$(330) \leftarrow(021)$ & 678.45 & 0.03 & 0.04 & -0.18 \\
\hline$(3111) \leftarrow(0210)$ & 675.22 & 0.03 & 0.00 & 0.10 \\
\hline$(3110) \leftarrow(029)$ & 695.92 & 0.03 & 0.04 & 0.10 \\
\hline$(331) \leftarrow(022)$ & 700.37 & 0.05 & 0.04 & -0.20 \\
\hline$\left(\begin{array}{ll}33 & 2\end{array}\right) \leftarrow\left(\begin{array}{ll}02 & 3\end{array}\right)$ & 723.08 & 0.05 & 0.05 & -0.21 \\
\hline$(318) \leftarrow(027)$ & 739.48 & 0.06 & 0.02 & -0.01 \\
\hline$(317) \leftarrow(026)$ & 762.50 & 0.06 & 0.04 & -0.04 \\
\hline
\end{tabular}

a Observed by Lees and Baker (l). 
TABLE V

Orserved and Calculated $Q$ Branch Origins of $\mathrm{CD}_{3} \mathrm{OD}$

\begin{tabular}{|c|c|c|c|c|}
\hline$\left(\mathrm{n}^{\prime} \tau^{\prime} \mathbf{K}^{\prime}\right) \leftarrow(\mathrm{n} \tau \mathrm{K})$ & $\nu_{\text {obsd }}\left(\mathrm{cm}^{-1}\right)$ & Acc. $\left(\mathrm{cm}^{-1}\right)$ & $\Delta_{1}\left(\mathrm{~cm}^{-1}\right)$ & $\Delta_{2}\left(r \mathrm{~m}^{-1}\right)$ \\
\hline$\left(\begin{array}{ll}03 & 1\end{array}\right) \leftarrow\left(\begin{array}{ll}01 & 0\end{array}\right)$ & $2.74007^{\mathrm{a}}$ & 0.00033 & -0.00026 & 0.00646 \\
\hline$\left(\begin{array}{ll}01 & 1\end{array}\right) \leftarrow\left(\begin{array}{ll}02 & 0\end{array}\right)$ & $0.07002^{\mathrm{a}}$ & 0.00033 & -0.00007 & -0.01136 \\
\hline$\left(\begin{array}{ll}02 & 2\end{array}\right) \leftarrow\left(\begin{array}{ll}03 & 1\end{array}\right)$ & $5.52600^{\mathrm{a}}$ & 0.00100 & 0.00098 & -0.00165 \\
\hline$\left(\begin{array}{ll}13 & 0\end{array}\right) \leftarrow\left(\begin{array}{ll}12 & 1\end{array}\right)$ & $1.62398^{\mathrm{a}}$ & 0.00033 & 0.00015 & -0.00757 \\
\hline$\left(\begin{array}{ll}31 & 1\end{array}\right) \leftarrow(120)$ & 418.65 & 0.03 & 0.05 & 0.18 \\
\hline$(337) \leftarrow(116)$ & 424.84 & 0.03 & -0.05 & 0.05 \\
\hline$(318) \leftarrow(027)$ & 429.48 & 0.03 & 0.13 & 0.53 \\
\hline$(436) \leftarrow(127)$ & 434.62 & 0.15 & -0.07 & 0.14 \\
\hline$\left(\begin{array}{ll}41 & 0\end{array}\right) \leftarrow\left(\begin{array}{ll}13 & 1\end{array}\right)$ & 442.37 & 0.15 & -0.08 & 0.05 \\
\hline$(317) \leftarrow(026)$ & 445.96 & 0.03 & 0.00 & 0.22 \\
\hline$\left(\begin{array}{ll}33 & 6\end{array}\right) \leftarrow\left(\begin{array}{ll}11 & 5\end{array}\right)$ & 447.79 & 0.03 & -0.05 & 0.00 \\
\hline$(437) \leftarrow(128)$ & 458.12 & 0.03 & 0.01 & 0.27 \\
\hline$(33 \quad 0) \leftarrow(021)$ & 458.02 & 0.15 & -0.07 & -0.09 \\
\hline$\left(\begin{array}{ll}41 & 1\end{array}\right) \leftarrow\left(\begin{array}{ll}13 & 2\end{array}\right)$ & 464.81 & 0.03 & 0.01 & 0.10 \\
\hline$(435) \leftarrow(114)$ & 470.95 & 0.03 & 0.04 & 0.06 \\
\hline$(331) \leftarrow(022)$ & 480.42 & 0.15 & 0.03 & -0.20 \\
\hline$(434) \leftarrow(113)$ & 494.33 & 0.03 & -0.05 & -0.06 \\
\hline$(4210) \leftarrow(139)$ & 499.43 & 0.03 & 0.03 & -0.20 \\
\hline$(3310) \leftarrow(019)$ & 517.37 & 0.03 & 0.00 & 0.14 \\
\hline$(429) \leftarrow(138)$ & 523.44 & 0.03 & 0.01 & -0.22 \\
\hline$(428) \leftarrow(137)$ & 549.17 & 0.03 & -0.01 & -0.24 \\
\hline$(338) \leftarrow(017)$ & 565.84 & 0.03 & -0.01 & 0.03 \\
\hline$(3213) \leftarrow-(1312)$ & 430.31 & 0.03 & 0.00 & -0.10 \\
\hline$(3313) \leftarrow(0112)$ & 455.01 & 0.03 & -0.03 & 0.30 \\
\hline$\left(\begin{array}{ll}31 & 6\end{array}\right) \leftarrow\left(\begin{array}{ll}02 & 5\end{array}\right)$ & 466.08 & 0.03 & 0.01 & 0.16 \\
\hline$(425) \leftarrow(234)$ & 555.38 & 0.03 & 0.02 & -0.75 \\
\hline$(337) \leftarrow\left(\begin{array}{ll}01 & 6\end{array}\right)$ & 591.85 & 0.03 & -0.03 & -0.05 \\
\hline$(325) \leftarrow(016)$ & 428.61 & 0.03 & -0.07 & -0.09 \\
\hline
\end{tabular}

a Observed by Lees (2).

the result, $L_{v}=0.09 \times 10^{-4} \mathrm{~cm}^{-1}$. Clearly our values for $D_{J J}, D_{J K}$ and $L_{v}$ are very approximate but fortunately the effect of these terms on the evaluation of the $Q$ branch origins turns out to be small.

In all 24 infrared $Q$ branch origins were found and these, together with the 4 microwave data ${ }^{5}$ were analyzed as before by nonlinear least squares and the resulting constants appear in Table I. The observed and calculated $Q$ branch origins are given in Table V. Again, the agreement is very satisfactory. Taking the 176 data points for all four isotopic molecules it is found that 72 or $41 \%$ of the $\Delta_{1}$ are less than the corresponding experimental accuracies.

${ }^{5}$ At the time our calculations were made Prof. Lees had determined $4 Q$ branch origins and these data he kindly furnished us and are listed in Tuble V. Recently he informed us that he has slightly revised his original values. The differences are, however, small and do not appear to warrant a recalculation of our analysis. 


\section{INTERRELATION OF 'THE KIRTMAN PERTURBATION CONSTANTS}

The foregoing analyses of the rotation-torsion spectra of the isotopic methanols is very satisfactory in many respects. With the aid of 40 parameters $(10$ for each molecule) the $176 Q$ branch origins have been computed and are found to agree very satisfactorily with their observed values. By using in addition the $a$-type parameters ( 8 for each molecule) as found by Lees and Baker from the microwave spectra it is possible to calculate the frequencies and intensities of all $P, Q$ and $R$ branch lines. More than 1000 of these can be identified in the infrared spectra as observed by Woods and l'eters. The agreement both as regards frequencies and intensities is very good and is of the order of the experimental accuracy. Moreover, there are no significantly strong lines in the measured spectra which are not accounted for.

The analyses are however unsatisfactory in two respects. In the first place the Kirtman perturbation constants appear as purely empirical parameters and are not easily understood in terms of the physical properties of the molecule. Secondly, as a consequence of the redundancy relation it is not possible to obtain what may be called the true values of the perturbation constants $V_{6}, k_{6}$ and $k_{7}$ nor the precise values of the large constants $I_{a 1}, I_{a 2}$, and $V_{3}$. It appears that the only way of resolving the difficulty is by interrelating the constants for the various isotopic molecules. The following considerations constitute such an approach although the resulting theory contains a number of approximations.

As a first step, which is introduced mainly for the purpose of simplifying the calculations, we will confine our attention to those energy levels of the molecule where the component of angular momentum along the symmetry axis is equal to the total angular momentum, that is, those states where $J=K$. If one now replaces $J^{2}+J$ in the energy expression by $K^{2}$ we will be describing a molecule where all rotations, including the hindered internal rotation, lie along the symmetry axis. In effect this reduces the original problem of 4 degrees of rotational freedom to one of 2 degrees of freedom.

Consider now those centrifugal distortion terms which are quartic in the matrix elements of angular momenta. Referring to Eq. (10) these are seen to be

$$
-D_{8} K^{4}+k_{1}{ }^{\prime} K^{3}\left\langle P_{\gamma}\right\rangle+k_{2}{ }^{\prime} K^{2}\left\langle P_{\gamma}{ }^{2}\right\rangle+k_{3} K\left\langle P_{\gamma}{ }^{3}\right\rangle+k_{4}\left\langle P_{\gamma}^{4}\right\rangle \text {, }
$$

where

$$
D_{s}=D_{J J}+D_{J K}+D_{K K}, k_{1}^{\prime}=k_{1}+L_{v} \text { and } k_{2}^{\prime}=k_{2}+G_{v}^{\prime} .
$$

In this description the coordinates are $\varphi$, the angle of rotation of the total molecule about the symmetry axis and $\gamma$, the angle of rotation of the hydroxyl group with respect to the methyl group. The corresponding momentum operators are $P_{\varphi}=(1 / i)(\delta / \delta \varphi)$ and $P_{\gamma}=(1 / i)(\delta / \delta \gamma)$. To a high order of approximation $P \varphi$ is represented by a diagonal matrix with the eigenvalues $K$. An alternate description of the two dimensional model can be made which employs 
the angles $\varphi_{1}$ and $\varphi_{2}$ measuring, respectively, the rotation of the hydroxyl and methyl groups relative to a fixed coordinate system. The corresponding angular momenta will be denoted by $P_{1}$ and $P_{2}$ and the relations between the two sets of coordinates and momenta are

$$
\begin{array}{ll}
\varphi=\alpha \varphi_{1}+\beta \varphi_{2}, & P_{\varphi}=P_{1}+P_{2}, \\
\gamma=\varphi_{1}-\varphi_{2}, & P_{\gamma}=\beta P_{1}-\alpha P_{2},
\end{array}
$$

where $\alpha=I_{a 1} /\left(I_{a 1}+I_{a 2}\right)$ and $\beta=1-\alpha$. A substitution yields the following expression for the quartic terms of the perturbation energy

$$
U_{1}\left\langle P_{1}^{4}\right\rangle+U_{2}\left\langle P_{1}^{3} P_{2}\right\rangle+U_{3}\left\langle P_{1}{ }^{2} P_{2}{ }^{2}\right\rangle+U_{4}\left\langle P_{1} P_{2}^{3}\right\rangle+U_{5}\left\langle P_{2}^{4}\right\rangle .
$$

The connections between the new and the old sets of constants are given by the formula

$$
\left[\begin{array}{l}
-D_{s} \\
k_{1}{ }^{\prime} \\
k_{2}{ }^{\prime} \\
k_{3} \\
k_{4}
\end{array}\right]=\left[\begin{array}{lllll}
\alpha^{4} & \alpha^{3} \beta & \alpha^{2} \beta^{2} & \alpha \beta^{3} & \beta^{4} \\
4 \alpha^{3} & \alpha^{2}(4 \beta-1) & 2 \alpha \beta(\beta-\alpha) & \beta^{2}(1-4 \alpha) & -4 \beta^{3} \\
6 \alpha^{2} & 3 \alpha(\beta-\alpha) & (1-6 \alpha \beta) & 3 \beta(\alpha-\beta) & 6 \beta^{2} \\
4 \alpha & (1-4 \alpha) & 2(\alpha-\beta) & (4 \beta-1) & -4 \beta \\
1 & -1 & 1 & -1 & 1
\end{array}\right]\left[\begin{array}{l}
U_{1} \\
U_{2} \\
U_{3} \\
U_{4} \\
U_{j}
\end{array}\right]
$$

The quartic perturbation terms are the analog of the usual type of centrifugal distortion terms which arise from the nonrigidity of the molecular framework. They are calculated by expanding the moments of inertia as a power series in the normal vibrational coordinates and introducing the vibrational potential energy. For a one degree of freedom rotator, first order perturbation theory yields a resulting term which is proportional to the expectation value of the fourth power of the angular momentum. The proportionality factor contains the potential energy constants and is inversely proportional to the square of the moment of inertia. In our case which, although more complicated, has been reduced to rotations about the symmetry axis, it is expected that the most important normal vibrations which produce these distortion terms will be those involving mainly the motions of the hydrogen or deuterium atoms. As a consequence of this assumption it would follow that $U_{1}$ could be written as $g_{1} U_{1}^{0}$, where $U_{1}^{0}$ is a constant common to all the isotopic methanol molecules and where the mass factor $g_{1}$ would equal 1 for $\mathrm{CH}_{3} \mathrm{OH}$ and $\mathrm{CD}_{3} \mathrm{OH}$ but would be $1 / 4$ for $\mathrm{CH}_{3} \mathrm{OD}$ and $\mathrm{CD}_{3} \mathrm{OD}$. By inverting $\mathrm{Eq}$. (22) to solve for $U_{1}$ this conjecture can be tested by substituting the constants appearing in Table I and using the values of $D_{J J}, D_{J K}, L_{v}$ and $G_{v}$ given by Lees and Baker. The resulting numerical values for $U_{1}$ are, respectively, $-79.09,-87.04,-23.49$ and $-25.09 \times 10^{-4} \mathrm{~cm}^{-1}$.

Although the predictions of this test are only approximately satisfied, the result is sufficiently encouraging to warrant further exploration. The generalization would consist in letting $U_{i}=g_{i} U_{i}^{0}, i=1,2,3,4,5$ where $g_{i}$ are the mass factors listed in the first 5 columns of Table VI. 
TABLE VI

Mass Factors in the Equation $U_{i}=g_{i} U_{i}{ }^{0}$

\begin{tabular}{lllllllll}
\hline & $g_{1}$ & $g_{2}$ & $g_{3}$ & $g_{4}$ & $g_{5}$ & $g_{6}$ & $g_{7}$ & $g_{8}$ \\
\hline $\mathrm{CH}_{3} \mathrm{OH}$ & 1 & 1 & 1 & 1 & 1 & 1 & 1 & 1 \\
$\mathrm{CH}_{3} \mathrm{OD}$ & $1 / 4$ & $1 / 4 \sqrt{2}$ & $1 / 2$ & $1 / 2 \sqrt{2}$ & 1 & $1 / 2$ & $1 / 2 \sqrt{2}$ & 1 \\
$\mathrm{CD}_{3} \mathrm{OH}$ & 1 & $1 / 2 \sqrt{2}$ & $1 / 2$ & $1 / 4 \sqrt{2}$ & $1 / 4$ & 1 & $1 / 2 \sqrt{2}$ & $1 / 2$ \\
$\mathrm{CD}_{3} \mathrm{OD}$ & $1 / 4$ & $1 / 4$ & $1 / 4$ & $1 / 4$ & $1 / 4$ & $1 / 2$ & $1 / 2$ & $1 / 2$ \\
\hline
\end{tabular}

In a similar manner one may treat the quadratic perturbation terms. These may be interpreted as measuring the change in barrier height $V_{3}$ caused by centrifugal distortions of the rotating molecule.

Again by letting $J=K$, these have the two forms

$$
\begin{aligned}
& k_{5}{ }^{\prime} K^{2}\langle 1-\cos 3 \gamma\rangle+k_{6} K\left\langle P_{\gamma}(1-\cos 3 \gamma)\right\rangle+k_{7}\left\langle P_{\gamma}{ }^{2}(1-\cos 3 \gamma)\right\rangle \text { or } \\
& U_{6}\left\langle P_{1}{ }^{2}(1-\cos 3 \gamma\rangle+U_{7}\left\langle P_{1} P_{2}(1-\cos 3 \gamma)\right\rangle+U_{8}\left\langle P^{2}(1-\cos 3 \gamma)\right\rangle,\right.
\end{aligned}
$$

where $k_{5}{ }^{\prime}=k_{5}+F_{v}$. The connections between the two sets of constants are

$$
\begin{aligned}
& k_{8}^{\prime}=\alpha^{2} U_{6}+\alpha \beta U_{7}+\beta^{2} U_{8}, \\
& k_{6}=2 \alpha U_{6}+(\beta-\alpha) U_{7}-2 \beta U_{8}, \\
& k_{7}=U_{6}-U_{7}+U_{8} .
\end{aligned}
$$

If we employ the assumption that the most important normal vibrations are those involving mainly the motions of the hydrogens, or deuteriums, we would let $U_{k}=g_{k} U_{k}^{0}, k=6,7,8$ with the mass factors listed in the last 3 columns of Table VI. As before $U_{k}{ }^{0}$ would be taken to be the same for all four isotopic molecules. Note that the constants $k_{5}{ }^{\prime}, k_{6}$ snd $k_{7}$ must be given what we call their true values. In particular, $k_{6}$ and $k_{7}$ are not equal to the quantities appearing in Table I since, as has been stated, the redundancy relation does not allow their unique evaluation from the data for any one of the isotopic molecules. Thus it is not possible to carry out the test for the behavior of, say, $U_{6}$ which was performed on $U_{1}$.

On the basis of the foregoing tentative theory we shall attempt to determine the constants using as data points the $176 Q$ branch origins observed for all four isotopic methanols. There will be 12 primary constants, namely, $I_{a 1}, I_{a 2}$, and $V_{3}$ for each molecule. There are eight centrifugal distortions parameters, $U_{1}^{0}, \cdots, U_{8}^{0}$. Finally there is the potential constant $V_{6}$ which measures the deviation from a sinusoidal barrier potential. Since $V_{6}$ is expected to be much smaller than $V_{3}$ it can be safely assumed that $V_{6}$ will have the same value, within the limits of experimental error, for each isotopic molecule.

The computed values of $D_{K R}$ which appear in Table $\mathrm{I}$ could have been employed as constraints upon the constants $U_{1} \cdots, U_{5}$ through the use of Eq. (22). 
However, it was considered better to introduce them as four additional data points with estimated accuracies of $1.0,0.6,0.3$ and $0.3 \times 10^{-6} \mathrm{~cm}^{-1}$, respectively. Thus in all there are 180 data points to be described with the aid of 21 parameters. This is in contrast to the earlier computations which employed 40 parameters to determine 176 data points.

An appropriate nonlinear least squares program was devised which, when applied, led to convergent values for the parameters after only a few iterations. These are listed in Table VII. The $Q$ branch origins were then recomputed and compared with the observed origins. The last columns in Tables II, III, IV and $\mathrm{V}$ list $\Delta_{2}=\nu_{\mathrm{obsd}}-\nu_{\mathrm{calcd}}$. An inspection of these tables shows that most of the $\Delta_{2}$ are definitely larger than $\Delta_{1}$. Only $18 \%$ of the $\Delta_{2}$ are less than the experimental accuracies whereas the corresponding figure for $\Delta_{1}$ was $41 \%$. This poorer agreement might perhaps have been expected since only about half as many parameters are employed. However, a more important reason probably stems from the approximations entering the tentative theory. A second method of comparing the degree of agreement may be made by computing the RMS of the standard deviation $S$, where $S$ is defined as ( $\nu_{\text {obsd }}-\nu_{\text {ealed }}$ ) divided by the experimental accuracy. $S$ is found to have the value 2.33 for the $\Delta_{1}$ and 9.07 for the $\Delta_{2}$. Ideally if the theory is exact and if the estimated experimental accuracies are correct the expected value of $S$ is 1 . (12)

Although the agreement with the measured spectrum is less good, the tentative theory possesses some real advantages in spite of its approximate character. Onc of the principal advantages is that the perturbation constants of all four isotopic molecules have been interrelated and consequently the limitations imposed by the redundancy relation have been eliminated. Thus the value of $V_{6}=-0.07 \pm 0.21$ should measure the true deviation from a sinusoidal

TABLE VII

ViLUes of THE 21 P.IRAMETERS

\begin{tabular}{|c|c|c|c|c|}
\hline \multirow{4}{*}{$\mathrm{CH}_{3} \mathrm{OH}$} & $I_{a 1}\left(10^{-40} \mathrm{gm} \mathrm{cm}^{2}\right)$ & $1.2721 \pm 0.0028$ & $V_{6}\left(\mathrm{~cm}^{-1}\right)$ & $-0.07 \pm 0.21$ \\
\hline & $I_{a 2}\left(10^{-40} \mathrm{gm}\left(\mathrm{cm}^{2}\right)\right.$ & $5.3082 \pm 0.0030$ & $U_{1}^{0}\left(10^{-4} \mathrm{~cm}^{-1}\right.$ & $-85.03 \pm 0.58$ \\
\hline & $V_{3}\left(\mathrm{~cm}^{-1}\right)$ & $372.29 \pm 0.21$ & $U_{2}^{0}\left(10^{-4} \mathrm{~cm}^{-1}\right)$ & $6.88 \pm 0.83$ \\
\hline & & & $U_{3}{ }^{0}\left(10^{4} \mathrm{~cm}^{1}\right)$ & $-0.76 \pm 0.86$ \\
\hline \multirow{4}{*}{$\mathrm{CH}_{2} \mathrm{OD}$} & $I_{a 1}\left(10^{-40} \mathrm{gm} \mathrm{cm}^{2}\right)$ & $2.3912 \pm 0.0047$ & $U_{4}{ }^{0}\left(10^{-4} \mathrm{~cm}^{-1}\right)$ & $1.09 \pm 0.32$ \\
\hline & $I_{a 2}\left(10^{-40} \mathrm{gm} \mathrm{cm}^{2}\right)$ & $5.2993 \pm 0.0041$ & $U_{5}{ }^{0}\left(10^{-4} \mathrm{~cm}^{-1}\right)$ & $-1.15 \pm 0.12$ \\
\hline & $V_{3}\left(\mathrm{~cm}^{-1}\right)$ & $364.95 \pm 0.13$ & $U_{6}{ }^{0}\left(\mathrm{~cm}^{-1}\right)$ & $0.419 \pm 0.048$ \\
\hline & & & $U_{\mathrm{\gamma}}^{0}\left(\mathrm{~cm}^{-1}\right)$ & $0.0175 \pm 0.0018$ \\
\hline \multirow{3}{*}{$\mathrm{CD}_{3} \mathrm{OH}$} & $I_{a 1}\left(10^{-40} \mathrm{gm} \mathrm{cm}^{2}\right)$ & $1.2762 \pm 0.0028$ & $U_{8}^{0}\left(\mathrm{~cm}^{-1}\right)$ & $-0.0140 \pm 0.0032$ \\
\hline & $I_{a 2}\left(10^{-40} \mathrm{gm}\left(\mathrm{cm}^{2}\right)\right.$ & $10.5754 \pm 0.0045$ & & \\
\hline & $V_{3}\left(\mathrm{~cm}^{-1}\right)$ & $368.24 \pm 0.22$ & & \\
\hline \multirow{3}{*}{$\mathrm{CD}_{3} \mathrm{OII}$} & $I_{a 1}\left(10^{-40} \mathrm{gm} \mathrm{cm}^{2}\right)$ & $2.3921 \pm 0.0047$ & & \\
\hline & $I_{a 2}\left(10^{-40} \mathrm{gm} \mathrm{cm}^{2}\right)$ & $10.5575 \pm 0.0088$ & & \\
\hline & $V_{3}\left(\mathrm{~cm}^{-1}\right)$ & $361.19 \pm 0.20$ & & \\
\hline
\end{tabular}


barrier potential. Our result indicates that the deviation is indeed very small for methanol. One way of expressing this fact is to take the ratio $V_{6} / V_{3}=-0.0002 \pm 0.0006$. It will be recalled that the crude electrostatic model for the methanol molecule gave $V_{6} / V_{3}=-0.0016$.

The values of $V_{3}$ are particularly interesting and show that the barrier height decreases slightly upon deuteration. A guide to the mechanism underlying this phenomenon is perhaps indicated by considering the electrostatic model. If account is taken of the zero point vibrations of the hydroxyl hydrogen and the methyl hydrogens one would obtain the barrier by calculating the mutual potential energy of these effective clouds of charge representing the probability distributions of the hydrogens in the staggered and eclipsed configurations. The barrier height would be equal to the difference between these two figures. Clearly since the potential is a very nonlinear function of the geometric dimensions the result will depend upon the shape and extent of the hydrogenic distributions and these will be altered by a deuterium substitution. Now the zero-point distributions can be expected to be mainly due to the vibrations of the hydrogens rather than, for example, to the $\mathrm{C}-\mathrm{O}$ vibration. Using this simple model one would conclude that the change in $V_{3}$ caused by deuteration of the hydroxyl hydrogen alone plus the change caused by deuteration of the methyl hydrogens alone should be equal to the change caused by complete deuteration. Referring to Table VII it is seen that in going from $\mathrm{CH}_{3} \mathrm{OH}$ to $\mathrm{CH}_{3} \mathrm{OD}, V_{3}$ decreases by $7.34 \mathrm{~cm}^{-1}$ while in going from $\mathrm{CH}_{3} \mathrm{OH}$ to $\mathrm{CD}{ }_{3} \mathrm{OH}, V_{3}$ decreases by $4.05 \mathrm{~cm}^{-1}$. The sum of these numbers is $11.39 \mathrm{~cm}^{-1}$ and the observed decrease of $V_{3}$ upon full deuteration is $11.10 \mathrm{~cm}^{-1}$.

As has been stated, the $D_{k \pi}$ for the various isotopic molecules were treated as four additional data points. The constants listed in Table VII lead to the following values for $D_{K K}: 0.36,0.27,0.10$ and $0.07 \times 10^{-4} \mathrm{~cm}^{-1}$, respectively. The agreement between these numbers and those appearing in Table $I$ is very good. It will be recalled that the latter were based upon an independent normal coordinate calculation.

Through the use of Eqs. (22) and (24) the parameters $U_{1}, \cdots, U_{8}$ can be transformed into the Kirtman perturbation constants $D_{K K}, k_{1}, \cdots, k_{7}$. The results appear in Table VIII. To the extent that the approximations of our tentative theory are valid, these Kirtman constants form unique sets which are not subject to the limitations of the redundancy relation Eq. (12). It is, however, also possible to obtain sets of constants which can be compared directly with the parameters given in Table I. These earlier values were calculated under the conditions of Fit II where $k_{7}$ was arbitrarily set equal to zero. The appropriate relations are given by Eqs. (13a) to (13e) and the results of the transformation are listed in Table IX.

An inspection of the numbers in Tables I and IX shows that while there is a great similarity between the values of the parameters, the majority of the in- 
TABLE VIII

"True" $b$-Type Parameters of the Isotopic Methanols

\begin{tabular}{lcccc}
\hline \multicolumn{1}{c}{ Parameters } & $\mathrm{CH}_{3} \mathrm{OH}$ & \multicolumn{1}{c}{$\mathrm{CH}_{3} \mathrm{OD}$} & \multicolumn{1}{c}{$\mathrm{CD}_{3} \mathrm{OH}$} & \multicolumn{1}{c}{$\mathrm{CD}_{3} \mathrm{OD}$} \\
\hline$I_{a 1}\left(10^{-40} \mathrm{gm} \mathrm{cm}^{2}\right)$ & 1.2721 & 2.3912 & 1.2762 & 2.3921 \\
$I_{a 2}\left(10^{-40} \mathrm{gm} \mathrm{cm}^{2}\right)$ & $\mathbf{5 . 3 0 8 2}$ & $\mathbf{5 . 2 9 9 3}$ & $\mathbf{1 0 . 5 7 5 4}$ & $\mathbf{1 0 . 5 5 7 5}$ \\
$V_{3}\left(\mathrm{~cm}^{-1}\right)$ & 372.29 & 364.95 & 368.24 & 361.19 \\
$V_{6}\left(\mathrm{~cm}^{-1}\right)$ & -0.07 & -0.07 & -0.07 & -0.07 \\
$D_{K K}\left(10^{-4} \mathrm{~cm}^{-1}\right)$ & 0.36 & 0.27 & 0.10 & 0.07 \\
$k_{1}\left(10^{-4} \mathrm{~cm}^{-1}\right)$ & $\mathbf{0 . 5 3}$ & -0.60 & $\mathbf{0 . 5 5}$ & 0.18 \\
$k_{2}\left(10^{-4} \mathrm{~cm}^{-1}\right)$ & -21.61 & -14.08 & -6.25 & -4.68 \\
$k_{3}\left(10^{-4} \mathrm{~cm}^{-1}\right)$ & -57.10 & -21.69 & -31.21 & -13.19 \\
$k_{4}\left(10^{-4} \mathrm{~cm}^{-1}\right)$ & -94.91 & -25.99 & -90.95 & -23.73 \\
$k_{5}\left(10^{-4} \mathrm{~cm}^{-1}\right)$ & 116.74 & 177.08 & 23.00 & 52.17 \\
$k_{6}\left(10^{-4} \mathrm{~cm}^{-1}\right)$ & 1953.52 & $\mathbf{1 5 2 4 . 7 4}$ & 1124.02 & 932.36 \\
$k_{7}\left(10^{-4} \mathrm{~cm}^{-1}\right)$ & 3877.28 & 1832.46 & 3998.47 & 1938.64 \\
\hline
\end{tabular}

TABLE IX

Fit II Values of the $b$-Type Parameters Derived from Table ViII

\begin{tabular}{lcccc}
\hline \multicolumn{1}{c}{ Parameters } & \multicolumn{1}{c}{$\mathrm{CH}_{3} \mathrm{OH}$} & $\mathrm{CH}_{3} \mathrm{OD}$ & \multicolumn{1}{c}{$\mathrm{CD}_{3} \mathrm{OH}$} & \multicolumn{1}{c}{$\mathrm{CD}_{3} \mathrm{OD}$} \\
\hline$I_{\alpha 1}\left(10^{-40} \mathrm{gm} \mathrm{cm}^{2}\right)$ & 1.2484 & 2.3466 & 1.2526 & 2.3518 \\
$I_{a 2}\left(10^{-4} \mathrm{gm} \mathrm{cm}^{3}\right)$ & 5.3320 & 5.3439 & 10.5990 & 10.5978 \\
$V_{3}\left(\mathrm{~cm}^{-1}\right)$ & 374.03 & 365.77 & 370.04 & 362.06 \\
$V_{6}\left(\mathrm{~cm}^{-1}\right)$ & -1.39 & -1.04 & -1.57 & -1.27 \\
$D_{K K}\left(10^{-4} \mathrm{~cm}^{-1}\right)$ & 0.36 & 0.27 & 0.10 & 0.07 \\
$k_{1}\left(10^{-4} \mathrm{~cm}^{-1}\right)$ & 0.53 & -0.60 & 0.55 & 0.18 \\
$k_{9}\left(10^{-4} \mathrm{~cm}^{-1}\right)$ & -21.61 & -14.08 & -6.24 & -4.68 \\
$k_{3}\left(10^{-4} \mathrm{~cm}^{-1}\right)$ & -57.10 & -21.69 & -31.21 & -13.19 \\
$k_{4}\left(10^{-4} \mathrm{~cm}^{-1}\right)$ & -94.91 & -25.99 & -90.95 & -23.73 \\
$k_{5}\left(10^{-4} \mathrm{~cm}^{-1}\right)$ & 116.75 & 177.08 & 23.00 & 52.16 \\
$k_{6}\left(10^{-4} \mathrm{~cm}^{-1}\right)$ & -17.55 & -488.53 & 145.52 & 23.04 \\
$k_{7}\left(10^{-4} \mathrm{~cm}^{-1}\right)$ & 0 & 0 & 0 & 0 \\
\hline
\end{tabular}

dividual constants of Table IX lie outside the probable errors listed in Table I. This circumstance may well be a consequence of applying probability theory to rituations where the theoretical formulas are inexact. In any case they are developments in orders of approximation with the neglect of higher order terms. Under these conditions the differences between the calculated and observed frequencies are not entirely due to accidental errors and hence the calculated probable errors may not be a very good measure of the accuracy with which the constants have been determined.

In summary we believe that the most reliable calculations of the torsionrotation energy levels of the various isotopic molecules of methanol will result from employing the parameters given in Table I. On the other hand we think 
that the best description of the physical properties of the molecules will require the use of the parameters in either Table VII or Table VIII.

\section{ACK NOWLEDGMENTS}

We would like to express our thanks to Prof. R. M. Lees for helpful discussions and for sending us the results of his microwave measurements of the $\mathrm{CD}_{3} \mathrm{OD}$ spectrum prior to publication. We are likewise very grateful to Dr. D. R. Woods and to Prof. C. W. Peters for furnishing us with copies of their infrared spectra during the course of their work and for their helpful interest in the present investigation. This research has been supported in part by the U. S. Atomic Energy Commission.

Received: February 18, 1972

\section{REFERENCES}

1. R. M. Lees And J. G. Baker, J. Chem. Phys. 48, 5299 (1968).

2. R. M. Lees, in press. J. Chem. Phys.

9. D. R. Woops, thesis, University of Michigan, (1970).

4. J. S. Koehler and D. M. Dennison, Phys. Rev. 67, 1004 (1940).

5. D. G. Burkhard and D. M. Dennison, Phys. Rev. 84, 408 (1951).

6. E. V. Ivash And D. M. Dennison, J. Chem. Phys. 21, 1804 (1953).

7. K. T. Hecht and D. M. Dennison, J. Chem. Phys. 26, 48 (1957).

8. D. G. Burkhard and D. M. Dennison, $J$. Mol. Spectosc. 3, 299 (1959).

9. B. Kirtman, J. Chem. Phys. 37, 2516 (1962).

10. C. C. Lin and J. D. Swalen, Rev. Mod. Phys. 31, 841 (1959).

11. M. Margottin-Maclou, J. Phys. el Rad. 21, 634 (1960); J. Chim. Phys. 63, 215 (1966).

12. E. R. Cohen and J. W. M. Du Mond, "Handbuch der Physik" (S. Flügge, Ed.), Vol. 35, p. 53, Springer-Verlag. Berlin, 1957. 